\title{
Survey of Highly Non-Keplerian Orbits With Low-Thrust Propulsion
}

\author{
Robert J. McKay ${ }^{1}$, Malcolm Macdonald ${ }^{2}$, James Biggs ${ }^{3}$, Colin McInnes ${ }^{4}$ \\ Advanced Space Concepts Laboratory, University of Strathclyde, Glasgow G1 1XJ, United Kingdom
}

\section{Nomenclature}

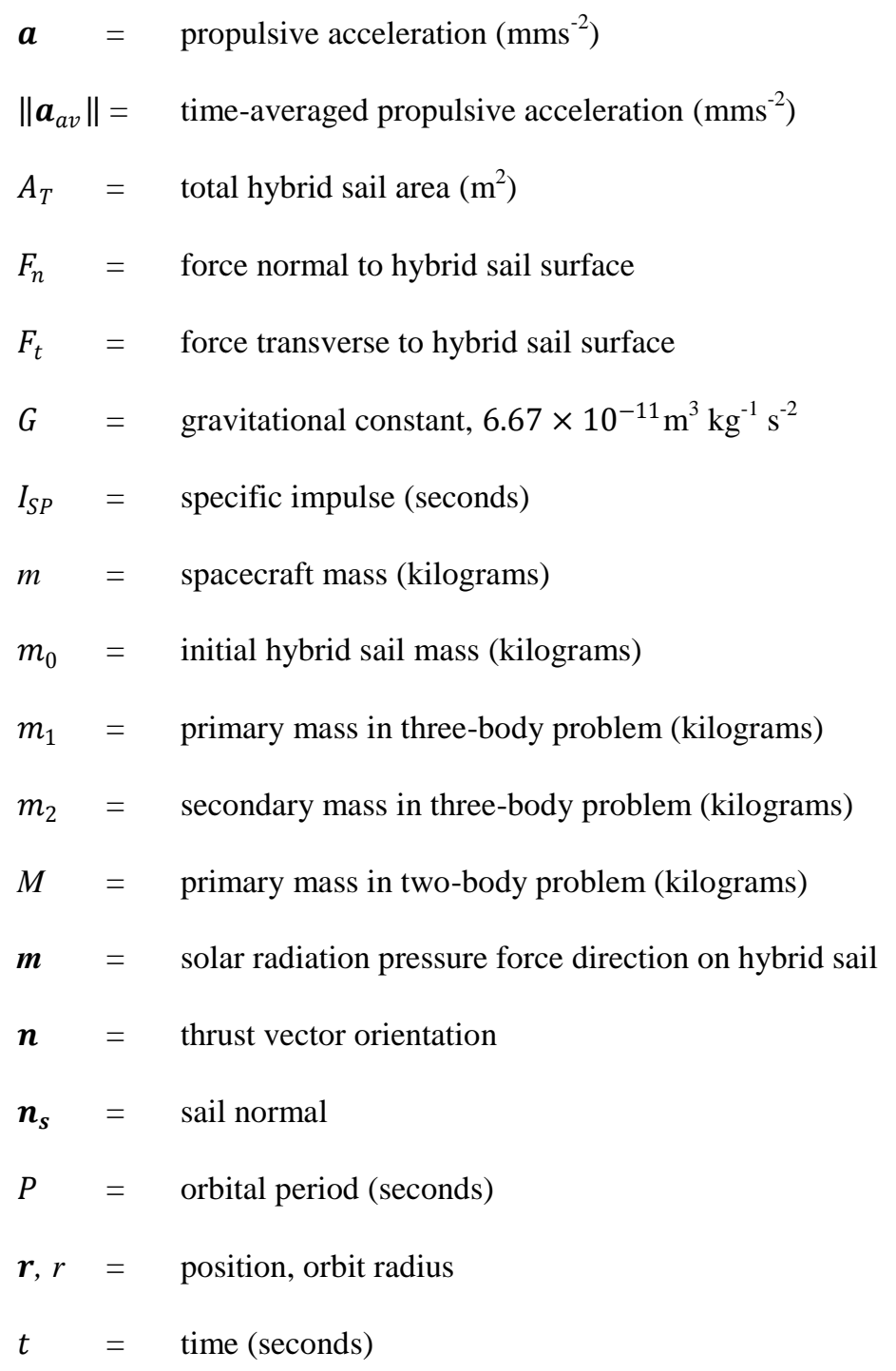

\footnotetext{
${ }^{1}$ Research Fellow, Advanced Space Concepts Laboratory (ASCL), University of Strathclyde, Glasgow, UK.

${ }^{2}$ Associate Director, ASCL, University of Strathclyde, Glasgow, UK, AIAA Associate Fellow.

${ }^{3}$ Associate Director, ASCL, University of Strathclyde, Glasgow, UK.

${ }^{4}$ Director, ASCL, University of Strathclyde, Glasgow, UK, AIAA Member.
} 


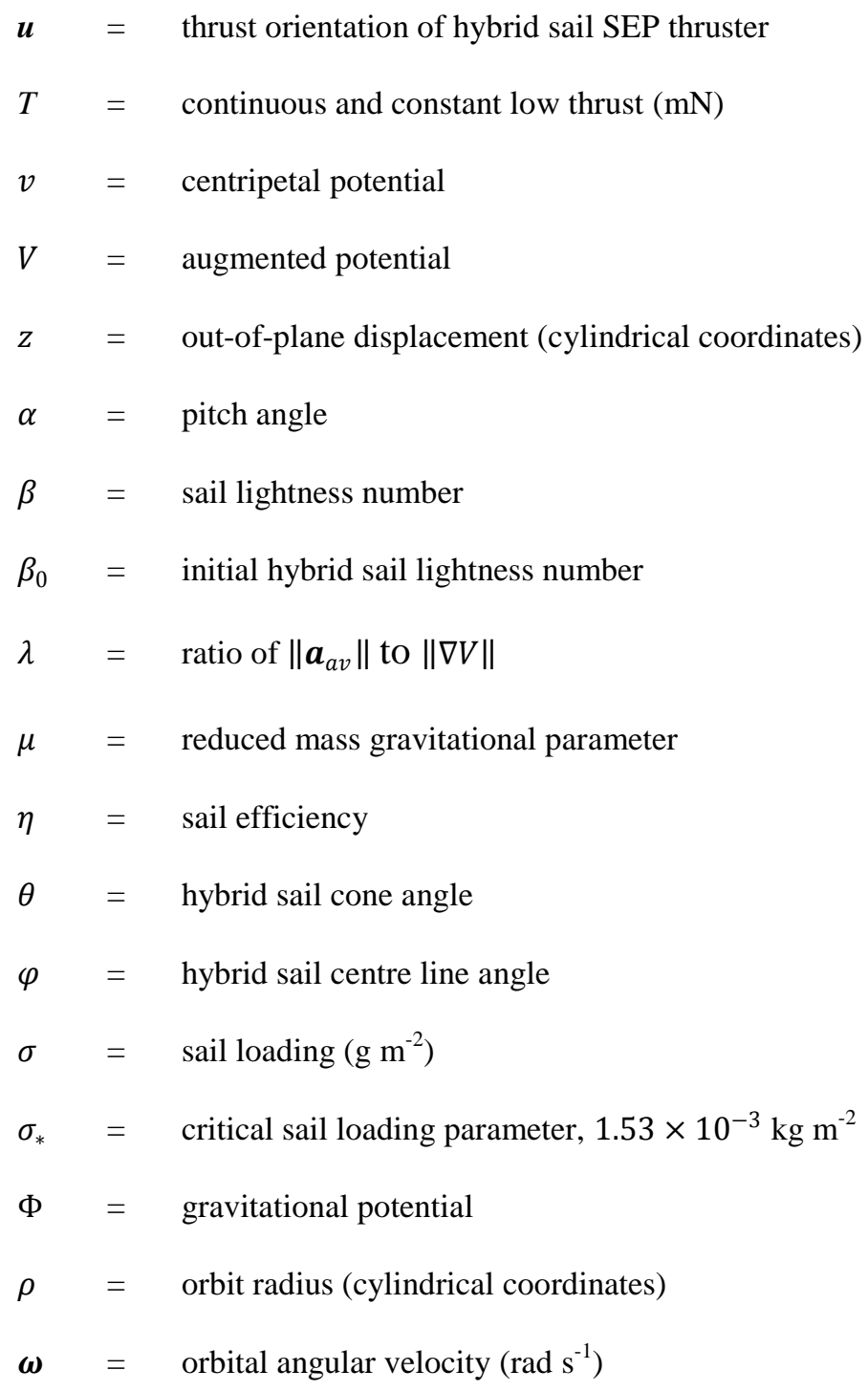

\section{Introduction}

$\mathrm{C}$ elestial mechanics has traditionally been concerned with orbital motion under the action of a conservative gravitational potential. In particular, the inverse square gravitational force due to the potential of a uniform, spherical mass leads to a family of conic section orbits, as determined by Isaac Newton, who showed that Kepler's laws were derivable from his theory of gravitation. While orbital motion under the action of a conservative gravitational potential leads to an array of problems with often complex and interesting solutions, the addition of non-conservative forces offers new avenues of investigation. In particular, non-conservative forces lead to a rich diversity of problems associated with the existence, stability and control of families of highly non-Keplerian orbits generated by a gravitational potential and a non-conservative force. 
Highly non-Keplerian orbits can potentially have a broad range of practical applications across a number of different disciplines. This review aims to summarize the combined wealth of literature concerned with the dynamics, stability and control of highly non-Keplerian orbits for various low thrust propulsion devices, and to demonstrate some of these potential applications.

The paper is structured as follows. In this section, firstly, a formal definition distinguishing the subset of highly nonKeplerian orbits from the larger sets of non-Keplerian orbits (NKOs) and natural Keplerian orbits is given. Then a brief historical overview of the literature on such orbits is given, tracing the field from its roots to current research. In Section 2 highly non-Keplerian orbits are examined for the two-body problem, which results in three distinct types of orbit, parameterized by the orbital period of the spacecraft. Orbit stability and control is discussed, as are connections between highly non-Keplerian two-body orbits. In Section 3 the discussion moves on to consider work pertaining to the three-body case, where the addition of a second large body results in a set of artificial equilibrium points (AEPs) being generated, when the angular velocity of rotation of the frame of reference is chosen to be that of the two primary masses. The literature on multiple different continuous low-thrust propulsion systems is considered, taking in solar electric propulsion (SEP), solar sails, hybrid SEP/sails and other more exotic methods. The literature on periodic orbits about displaced artificial equilibrium points high above the ecliptic plane, analogous to the classical "halo" orbits, is reviewed, as is the work on connections between highly non-Keplerian three-body orbits. Finally, extensions to the model are discussed briefly. Section 4 looks at some of the applications of highly nonKeplerian orbits suggested within the literature, covering broad areas such as telecommunications, planetary science and geoengineering, amongst others, whilst Section 5 provides the conclusions, with a summary of the paper as a whole and some points for the future.

\subsection{Defining highly non-Keplerian orbits}

Given the comparative simplicity of Kepler's laws, the set of non-Keplerian orbits can be deemed quite significant in its breadth and depth: Keplerian orbits neglect atmospheric drag, solar radiation pressure, non-spherical central bodies, and other perturbations. Indeed, perturbations pertaining to gravitational interactions with other objects are neglected in the Keplerian treatment, meaning that a spacecraft orbiting in the Sun-Earth system is technically nonKeplerian. However, historically, the term "non-Keplerian" has been used in reference to orbits where a perturbing or propulsive acceleration acts in addition to that of the effects of gravity (and so perhaps, in the context of threebody orbits, a better term might be natural orbits, where natural would refer to the consideration of only gravitational effects from a point mass - but such technicalities are considered an unnecessary diversion here). Thus, for the avoidance of doubt in this review paper, orbits that take into account perturbations other than gravity, such as solar radiation pressure and artificial propulsion methods, are formally termed as non-Keplerian orbits. A special subset of that set would then be the highly non-Keplerian orbits, where the use of the term "highly" will be defined subsequently, but, simply put, refers to the magnitude of the perturbing force. 
As stated, in order to have a non-Keplerian object, some additional accelerating/propelling force (beyond simply gravity from a point mass) is required. For a highly non-Keplerian orbit, the time-average of this acceleration over a full orbit is considered to be of at least equal magnitude to, if not greater than, that of the sum of the gravitational and centripetal accelerations experienced by the object (as viewed in a reference frame rotating at a given angular velocity relative to an inertial reference frame). Introducing a parameter $\lambda$ to represent this ratio, then $\lambda=$ $\left\|\boldsymbol{a}_{a v}\right\| /\|\nabla V\| \geq 1$, for highly non-Keplerian orbits, where $\left\|\boldsymbol{a}_{a v}\right\|$ is the time-average of the magnitude of the propulsive acceleration over one complete highly non-Keplerian orbit, and $\|\nabla V\|$ is the magnitude of the gravitational and centripetal accelerations experienced by the object. Even with such a careful definition, the authors do not consider this definition to be "fool-proof" - by which it is meant that, in the course of this paper orbits with $\lambda \gg 1$ are not considered (if such orbits even exist). For the purposes of this review, $\lambda$ may be greater than exactly 1 but is still approximately of order 1 .

Hence, with the parameter $\lambda$ providing the definition, orbits with $\lambda=0$ represent simple Keplerian orbits - in essence the large subset of classical celestial orbital mechanics without the addition of a non-conservative force. Orbits with $\lambda \ll 1$ (but nevertheless finite) are, in effect, weakly-perturbed Keplerian orbits, and as such may be referred to as non-Keplerian orbits. There are numerous examples of such orbits: for instance, there is a considerable body of work which has considered the use of rather small solar sails to artificially precess Earth-centered elliptical orbits for space physics mission applications. Macdonald and McInnes [1] showed that an ellipse will be precessed if the sail is Sun-facing, leaving the orbit averaged semi-major axis and eccentricity unchanged. By an appropriate choice of sail loading, the elliptical orbit can be forced to precess at a Sun-synchronous rate, maintaining a science payload permanently within the geomagnetic tail, a concept utilized by the GeoSail mission [2, 3, 4]. An even more recent example of such orbits is the extended Sun-synchronous orbits proposed by Macdonald et al. [5]. A separate example of non-Keplerian orbits would be those of the open spiral trajectories used for spacecraft transfers. It is worth remembering, however, that although these examples are of, in strict terms, non-Keplerian orbits, they are a quite separate subset of orbits to those considered in this review.

Highly non-Keplerian orbits can be obtained by considering the dynamics of a low thrust spacecraft in a rotating frame of reference, where the angular velocity of rotation of the frame of reference is used as a free parameter of the problem. Stationary solutions to the equations of motion can then be sought in this rotating frame of reference, which correspond to periodic, displaced orbits when viewed from an inertial frame of reference. In most situations, the instantaneous value of the propulsive acceleration required to maintain such an orbit is constant over that full orbit, and satisfies the requirement that $\lambda \geq 1$. In some circumstances, the instantaneous value of the propulsive acceleration required to maintain this orbit may vary (and as such may give an instantaneous value for $\lambda$ of less than 1 ), but over a complete orbit the time average value of the acceleration will ensure the condition $\lambda \geq 1$ is met. The text of the paper will make clear in each case how the requirement for highly non-Keplerian orbits is satisfied. 
Figure 1 thus sums up the sets of orbits as described in this subsection, with the division between the set of Keplerian orbits and the set of non-Keplerian orbits (and the further subset of highly non-Keplerian orbits) being made according to whether an additional perturbing or propulsive acceleration is present or not.

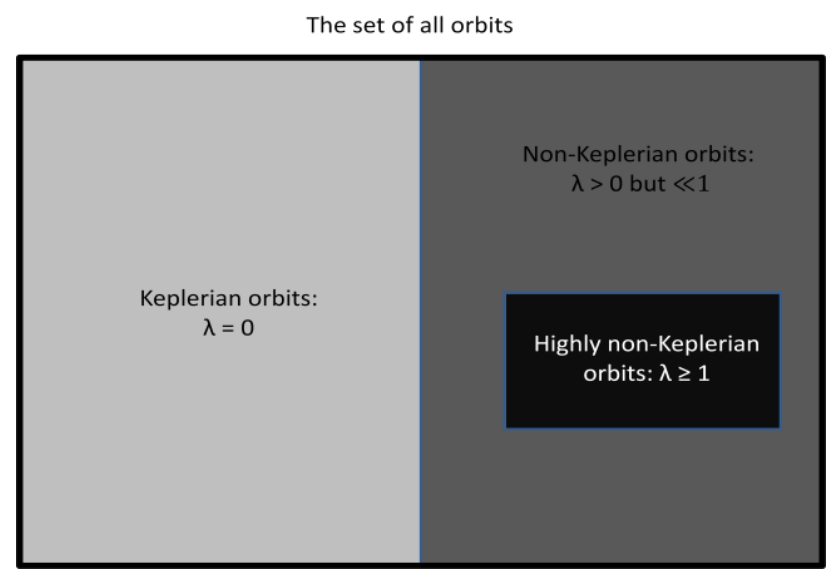

Fig. 1: Distinguishing between Keplerian, non-Keplerian, and highly non-Keplerian orbits. The box represents all possible orbits, of which Keplerian and non-Keplerian orbits are separate subsets thereof, and highly non-Keplerian orbits are a subset of the latter.

\subsection{Historical overview of non-Keplerian orbits}

Interestingly as early as 1929, Oberth, in a study of Earth orbiting reflectors for surface illumination [6], noted that solar radiation pressure will displace a reflector in a polar orbit in the anti-Sun direction. The implication of this is that the orbit plane does not contain the centre-of-mass of the Earth. Much later, the concept of counter-acting gravity through a propulsive thrust was apparently first formally suggested by Dusek [7], who pointed out that a spacecraft could be held in an artificial equilibrium at a location some distance from a natural libration point if the differences in gravitation and centripetal force (gravity gradient) were compensated for by continuous low thrust propulsion. Around a decade later, Austin et. al. [8] noted that propulsive thrust can in principle be used to displace the orbit of an artificial body, although only small displacements were considered for spacecraft proximity operations and no analysis of the problem was provided.

In the late 1970's/early 1980's Driver [9], outlined an analysis for a spacecraft that would hover directly above the poles of the Earth for an extended period of time, having apparently been inspired by an idea from the late $19^{\text {th }}$ century science fiction novel "Two Planets" (translated from the original "Auf Zwei Planeten" to English in the 1970's [10]). Such a "polesitter" concept would be enabled by continuous propulsion, where the thrust pointing direction was always diametrically opposite the direction of the perturbing acceleration acting on the spacecraft. Driver also indicated how the technology of the time could maintain a polesitter spacecraft using solar electric propulsion (SEP) for one or two years at several lunar distances from the Earth, and used to make continuous measurements of the Earth's polar regions. Similarly, Nock [11] noted that propulsive thrust can in principle be used 
to displace the orbit of an artificial body above, and parallel to, Saturn's rings for in-situ observations, but again no analysis of the problem was provided. At about the same time Forward considered using a solar sail to displace a body north or south of the geostationary ring $[12,13]$, which was around a decade after Farquhar had previously looked at using a small solar sail to stabilize motion near the classical $\mathrm{L}_{1}$ point in the Earth-Moon system [14]. By then the concept of solar sailing was already in itself not new - in 1921 Tsiolkovsky suggested that spacecraft could be propelled through space by using sunlight [15], and Tsander published the first practical paper on solar sailing in 1924 [16] - but it is only more recently that the necessary advances in materials have occurred and begun to make solar sail spaceflight look realistically achievable.

With such advances in mind, and following the work of Forward, Colin McInnes (hereby referred to simply as McInnes) made an extensive study of the concept of displaced orbits for solar sailing, exploring new regions of interest. This study began with McInnes \& Simmons analysis of halo orbits for solar sails [17], before moving on to consideration of displaced heliocentric orbits [18], which was apparently the first publication to investigate the full problem for such orbits. Subsequent work explored stability and control issues associated with such orbits in some detail, with it being demonstrated that the unstable families of displaced heliocentric orbits can be rendered linearly stable by a simple passive control law [19]. Optimum control methods have also been explored to generate minimum-time trajectories from Earth escape to displaced, non-Keplerian orbits using a combination of sequential quadratic programming and genetic algorithms [20]. Genetic algorithms are used to find a starting solution for the sequential quadratic programming algorithm, since transfers to highly non-Keplerian orbits are rather non-intuitive. A closed form solution for transfers to static equilibria, where the solar radiation pressure force exactly balances the solar gravitation force has also been found [21].

More work by McInnes \& Simmons extended the families of displaced heliocentric orbits to displaced planetcentred orbits [22]. These families require that the solar sail orbit plane is normal to the Sun-planet line. It is shown that new families of closed orbits are again generated, parameterised by the orbit period, while linearly stable and unstable families of orbits are also identified. It can be shown that the problem of a planet-centred solar sail orbit in fact reduces to the Stark problem (hydrogen atom in a uniform electric field [23]), if the sail orientation and Sun-line are both fixed. It has also been shown that the problem can be solved in closed form if the Sun-line is allowed to rotate [24]. A simpler problem, where the solar radiation pressure is directed along an assumed fixed Sun-line was considered by Dankowicz [25] for dust particles using the K-S transformation (see e.g. [26]). The results duplicated those found earlier by McInnes \& Simmons, however the use of K-S variables allows a non-linear stability analysis to be performed. Interestingly, the non-linear stability criteria derived are identical to the linear stability criteria identified in Ref. [22]. Additionally, Molostov and Shvartsburg [27, 28] and Mashkevich and Shvartsburg [29], considered the same set of problems as those in Ref. [22], but with a non-ideal solar sail (as will be seen later, the inclusion of a realistic force model for the solar sail can have a significant effect on the regions of existence of highly non-Keplerian orbits). 
A further extension of the work described above, a restricted three-body analysis has been performed which is shown to generate large sets of new artificial equilibria. The investigation of the existence and stability of new artificial Lagrange points in classical celestial mechanics has been pursued by several authors, mainly with application to interplanetary dust - the so-called photo-gravitational three-body problem. Chernikov [30], Schuerman [31] and Simmons et al. [32] have all considered this problem in some detail, while Murray [33] has considered the problem with generalized external forces. In particular Simmons et al. considered the case where the two primary masses are luminous, demonstrating the existence of a total of 9 Lagrange points [32]. The existence and stability of these equilibria are a function of the ratio of the mass and luminosity of the primaries.

The analysis discussed above for spherical dust particles has been extended to flat solar sails for both an ideal and non-ideal sail, with McInnes et al. presenting the first complete analysis of the problem [34]. Forward [35] also presented an approximate analysis of the problem based on a pre-print of Ref. [34], in the process coining the term "statite" in reference to a mission using a solar sail to hover above (or below) the Earth in such a displaced orbit. Such a concept has become generally known as a Polar Observer or PoleSitter mission (discussed further in Section 3.2), although as discussed above it appears the "polesitter" terminology had already been coined by Driver a few years earlier, albeit in the context of solar electric propulsion rather than solar sailing. It is shown in Ref. [34] that rather than singular equilibrium points, extended equilibrium surfaces are formed, parameterised by the sail loading (the mass per unit area of the sail assembly). A stability analysis has shown that these artificial equilibria are in general unstable, with stability only possible if the sail is Sun-facing, so that the problem is reduced to that investigated by Schuerman [31]. An important extension of the solar sail three-body problem was made in Ref. [36], which revises the original analysis for the case of a non-ideal solar sail. It had been assumed that this would have negligible influence on the volume of space within which artificial equilibria are allowed. However, even a small deviation from the ideal case is found to lead to a significant change to the boundary surfaces which delineate the regions of existence of artificial equilibria, this finding having serious practical implications which will be outlined later. McInnes collated a significant wealth of material on solar sailing and non-Keplerian orbits for a book on the subject [37], and the study of solar sail-enabled artificial displaced Lagrange points was considered extensively by NASA/JPL/NOAA under the GeoStorm mission concept in the late 1990's [38, 39].

In addition to highly non-Keplerian orbits for solar sails, large families of orbits are also found to exist for artificial bodies equipped with other forms of low thrust propulsion: more detail on such propulsion systems and orbit families is outlined in later Sections. While the use of low thrust propulsion in proximity to the Lagrange points of the restricted three-body problem has been considered by Dusek [7], resulting in displaced artificial equilibria as discussed earlier, large families of displaced orbits were first detailed by McInnes [40, 41]. It should be noted that the case of a displaced orbit where the thrust vector is aligned with the axis of symmetry of the orbit is equivalent to the case of a planet-centered solar sail orbit, where the sail is Sun-facing. This problem is contained in Ref. [22], and has also been considered by Dankowicz [25]. The orbits detailed by McInnes are again generated by considering the dynamics of the problem in a rotating frame of reference. Since the angular velocity of the frame of reference is 
used as a free parameter of the problem, the orbits can be classified into families by the functional form taken by the angular velocity. In particular, the required thrust induced acceleration can be minimized by an optimum selection of the angular velocity. Further analysis yields linear stability criteria which again provide necessary, but not sufficient conditions for stability. Ref. [41] also notes that individual displaced orbits can be patched together, or with Keplerian orbits, forming complex new trajectories - more discussion on this is included in Section 2.

\section{The Two-Body Case}

As discussed previously, various authors have noted the existence of displaced circular two-body orbits using low thrust propulsion. However it was McInnes who formally presented the parameterization of such orbits into three different types, dependent on their orbital period, in his consideration of the dynamics and stability of the orbits arising from the consideration of the two-body problem with additional thrust induced acceleration [40], and latterly the control of such orbits [41]. Consideration of the dynamics of a spacecraft with active propulsion generating continuous and constant acceleration $\boldsymbol{a}$ (via thrust $T$ in direction $\boldsymbol{n}$ ), in a frame of reference rotating with angular velocity $\boldsymbol{\omega}$ (relative to an inertial reference frame) about a body with gravitational potential $\Phi=-G M / r$, provides the conditions for stationary solutions to the equations of motion. Defining an augmented potential $V=\Phi+v$, with $v$ representing the centripetal acceleration, and using a set of cylindrical polar coordinates $(\rho, z)$, then the potential can be written as

$$
V(\rho, z ; \omega)=-\left[\frac{1}{2}(\omega \rho)^{2}+G M / r\right]
$$

and the required thrust-induced acceleration and thrust orientation vector (defined by a single pitch angle $\alpha$ ) are given by

$$
a(\rho, z ; \omega)=\left[\rho^{2}\left(\omega^{2}-\omega_{*}^{2}\right)^{2}+z^{2} \omega_{*}^{4}\right]^{1 / 2}
$$

and

$$
\tan \alpha(\rho, z ; \omega)=(\rho / z)\left[1-\left(\omega / \omega_{*}\right)^{2}\right]
$$

respectively, where

$$
\omega_{*}^{2}=\frac{G M}{r^{3}} .
$$


Given these conditions the spacecraft is stationary in the rotating reference frames and thus describes a circular orbit, displaced above the central body, as viewed from the inertial reference frame and shown in Figure 2.

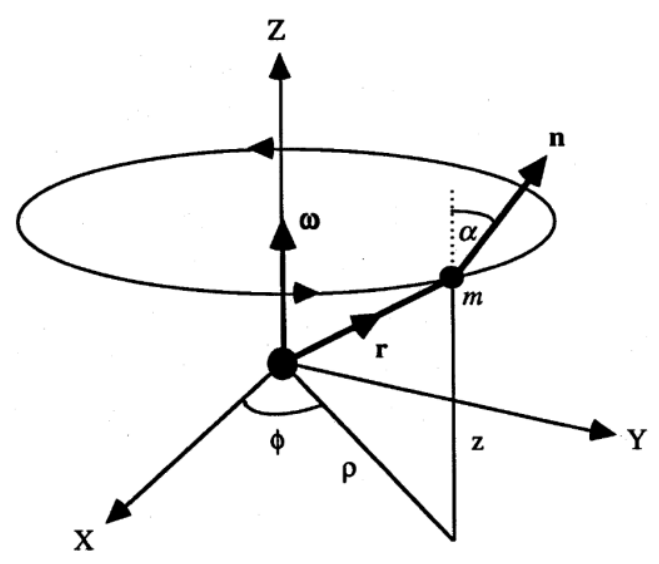

Fig. 2: Displaced orbit with thrust induced acceleration. From Ref. [41].

Having detailed the general conditions for displaced two-body orbits, three individual types or families can now be defined for the general case of a non-orientation constrained low-thrust propulsion system. For details on the families of two-body orbits found for the case of the solar sail (which, naturally, cannot thrust towards the Sun), see Ref. [37] for Sun-centered two-body non-Keplerian orbits and Ref. [42] for planet-centered non-Keplerian orbits.

\subsubsection{Type I}

If the orbit period is chosen such that $\omega=\omega_{*}$, i.e. the orbit period is fixed for a given value of $r$, then from Equation (9) the required thrust-induced acceleration is at its global minimum, and Equations (2) and (3) reduce to

$$
a=\frac{G M z}{r^{3}}
$$

and

$$
\tan \alpha=0
$$

respectively. This is the orbit family discussed by Dankowicz [25], and is essentially the same as that discussed previously in Ref. [22]. This family of orbits is exactly equivalent to the Stark problem with a hydrogen atom in a uniform electric field. With this value of propulsive acceleration, which does not vary over the course of the orbit, the parameter $\lambda$ is exactly equal to 1 . The required thrust-induced acceleration Type I orbit contours are 
demonstrated below, in Figures 3 and 4: Figure 3 illustrates the problem in a general non-dimensional context, with the axes being non-dimensional with respect to the radius of the central body and the acceleration contours being non-dimensional with respect to the gravitational acceleration at unit radius [41], and Figure 4 provides a more specific, re-dimensionalized example of the contours of a $1000 \mathrm{~kg}$ solar electric propulsion spacecraft with a maximum thrust of $300 \mathrm{mN}$ in the vicinity of Mars [43].

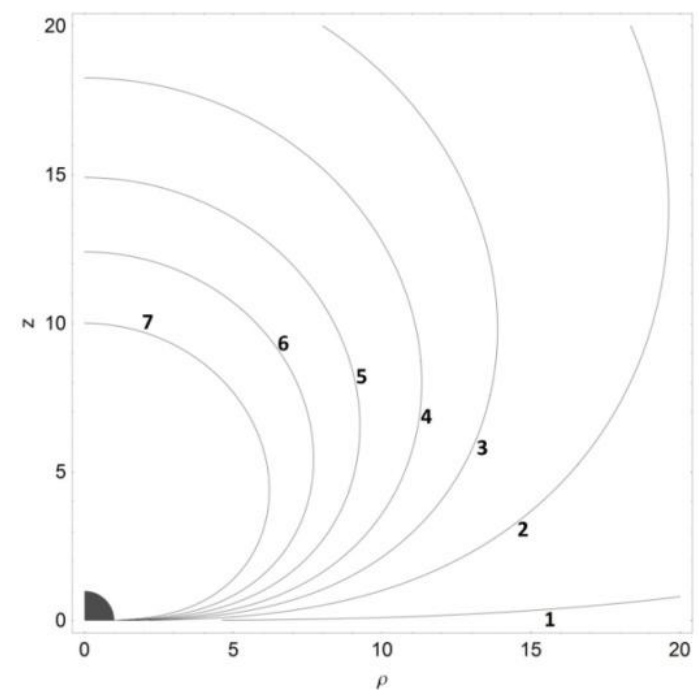

Fig. 3: Non-dimensional acceleration contours for Type I orbits, by contour: [1] $10^{-4} ;[2] 10^{-3} ;[3] 2 \times$ $10^{-3} ;[4] 3 \times 10^{-3} ;[5] 4.5 \times 10^{-3} ;[6] 6.5 \times 10^{-3} ;[7] 10^{-2}$. From Ref. [41].

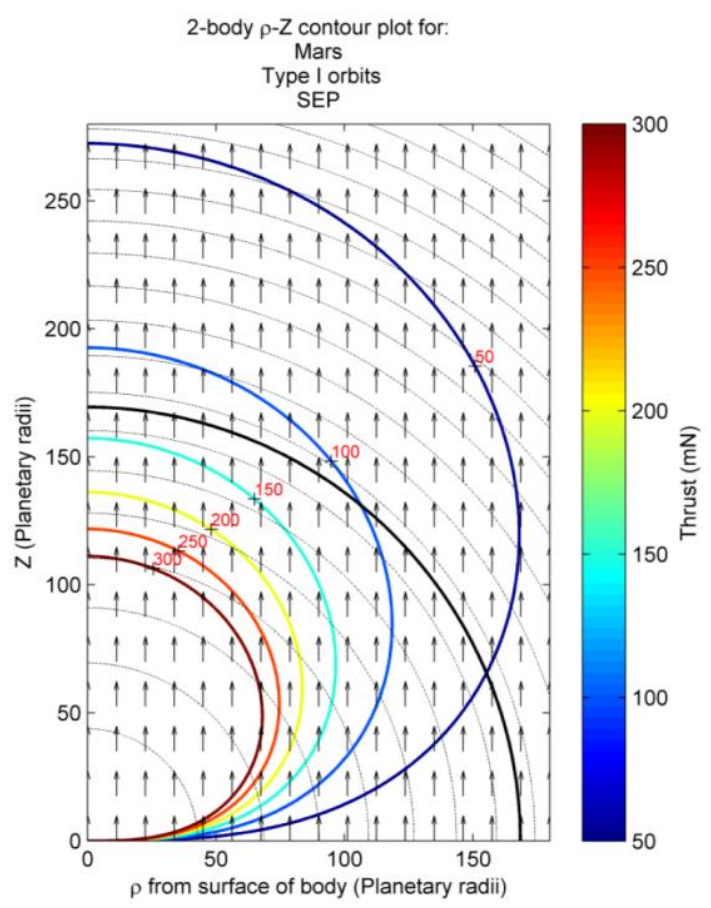

Fig. 4: Contours of constant thrust for Type I orbits about Mars using $1000 \mathrm{~kg}$ solar electric propulsion spacecraft with $300 \mathrm{mN}$ of thrust. The arrows represent the required thrust orientation to enable the orbit, the 


\section{thin dotted lines represent contours of constant orbital period, and the thick black line represents an approximation of the sphere of influence boundary of Mars. From Ref. [43].}

Considering Figure 4, it should of course be remembered that as the SEP system is active and burning propellant, the mass of the spacecraft will decrease over time. If the thrust level of the system is constant then this results in the acceleration of the spacecraft increasing over time, meaning that the spacecraft occupies new artificial equilibrium

points. The contours of Figure 4 therefore represent a snapshot for the case where the spacecraft mass is $3000 \mathrm{~kg}$, as opposed to a fixed point for all time. In practice either the level of the thrust from the SEP system would have to be throttled down to maintain the same artificial equilibrium point (which presently cannot be done in sufficiently small quantities, nor without continuity of thrust), or the spacecraft will move away from a given AEP over time. Provided the amount of propellant used is small, however, then Figure 2 provides a reasonable approximation of the motion of the spacecraft.

\subsubsection{Type II}

If the orbit period is chosen such that $\omega=\sqrt{G M / \rho^{3}}$, i.e. the orbit period is fixed for a given value of $\rho$, then Equations (2) and (3) become

$$
a=\left(\frac{G M}{r^{2}}\right)\left[1+\left[1+\left(\frac{z}{\rho}\right)^{2}\right]^{2}\left[1-2\left[1+\left(\frac{z}{\rho}\right)^{2}\right]^{-3 / 2}\right]\right]^{1 / 2}
$$

and

$$
\tan \alpha=\left(\frac{\rho}{z}\right)\left[1-\left[1+\left(\frac{Z}{\rho}\right)^{2}\right]^{3 / 2}\right]
$$

respectively, and a family of orbits synchronous with a body on a circular Keplerian orbit in the $z=0$ plane with the same orbit radius is generated. With this value of propulsive acceleration, which does not vary over the course of the orbit, the parameter $\lambda$ is greater than 1: the additional acceleration the spacecraft has compared to the Type I orbits allows the spacecraft to be displaced to different regions away from the central body, as can be seen from Figures 5 and 6, which show the Type II orbit contours in the same way as Figures 3 and 4 respectively. 


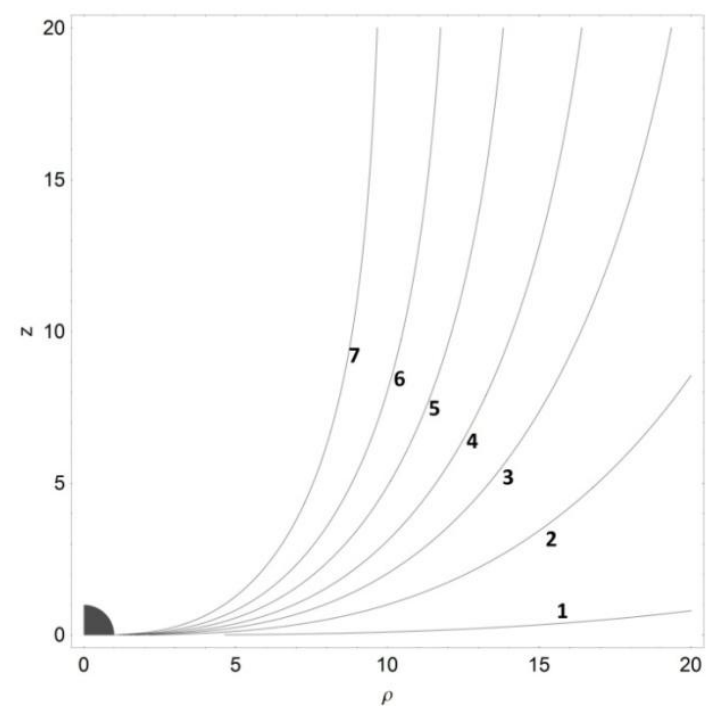

Fig. 5: Non-dimensional acceleration contours for Type II orbits, by contour: $[1] 10^{-4} ;[2] 10^{-3} ;[3] 2 \times$ $10^{-3} ;[4] 3 \times 10^{-3} ;[5] 4.5 \times 10^{-3} ;[6] 6.5 \times 10^{-3} ;[7] 10^{-2}$. From Ref. [41].

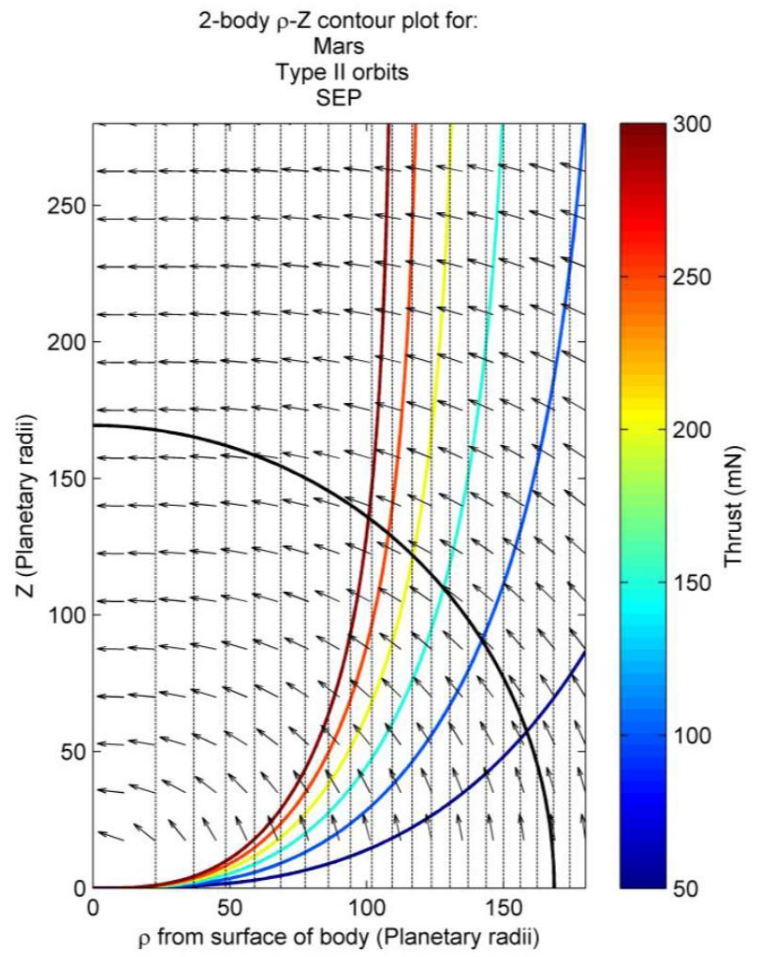

Fig. 6: Contours of constant thrust for Type II orbits about Mars using 1000kg SEP spacecraft with 300mN of thrust. From Ref. [43].

\subsubsection{Type III}

If instead the orbital period of the spacecraft is chosen such that it is constant throughout the entire $\rho-z$ plane, i.e. $\omega=\omega_{0}$, the acceleration and thrust direction equations become 


$$
a=\left[\rho^{2}\left(\omega_{0}^{2}-\omega_{*}^{2}\right)^{2}+z^{2} \omega_{*}^{4}\right]^{1 / 2}
$$

and

$$
\tan \alpha=\left(\frac{\rho}{z}\right)\left[1-\left(\frac{\omega_{0}}{\omega_{*}}\right)\right]^{2}
$$

respectively. Then a value of $\omega=\omega_{0}$ can be chosen such that the displaced orbits are synchronous with a Keplerian orbit with either a specific orbital radius $\rho$, or a specific orbital period $P$, given that

$$
\left(\frac{P}{2 \pi}\right)^{2}=\frac{r^{3}}{G M}=\frac{1}{\omega_{*}^{2}} .
$$

This results in two distinct branches of solutions corresponding to orbits in the $z=0$ plane or orbits displaced above this plane. Again, like the Type II orbits, with this value of propulsive acceleration, the parameter $\lambda$ is greater than 1. Figure 7 displays the non-dimensional thrust-induced acceleration contours for a value of $\omega_{0}$ chosen such that the displaced orbits are synchronous with a Keplerian orbit with radius $\rho=10$. Figure 8 shows the redimensionalized equithrust contours for the same SEP spacecraft about Mars as considered in Figs 4 and 6, synchronized with a Keplerian orbit of radius $\rho=110$ Mars radii.

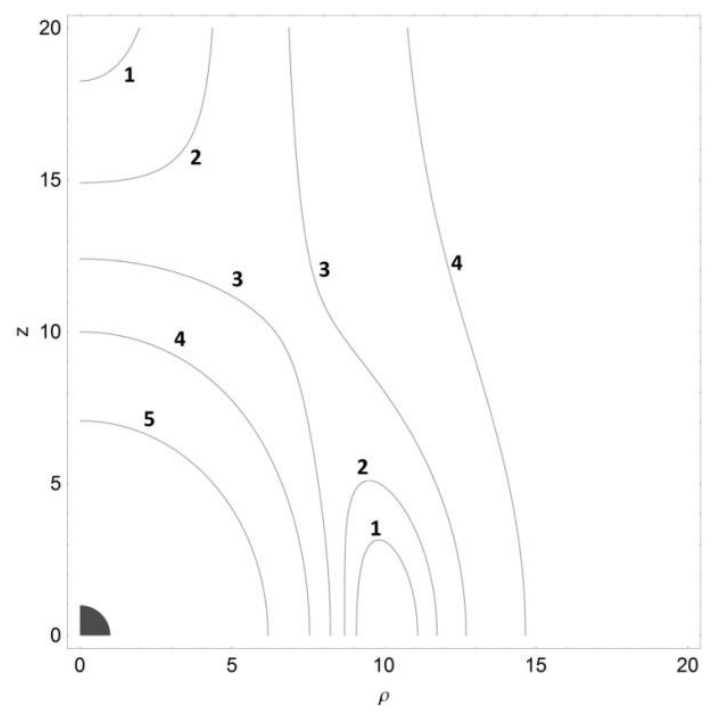

Fig. 7: Non-dimensional acceleration contours for Type III orbits, by contour: $[1] 3 \times 10^{-3} ;[2] 4.5 \times 10^{-3}$; [3] $6.5 \times 10^{-3} ;$ [4] $1 \times 10^{-2} ;$ [5] $2 \times 10^{-2}$. From Ref. [41]. 


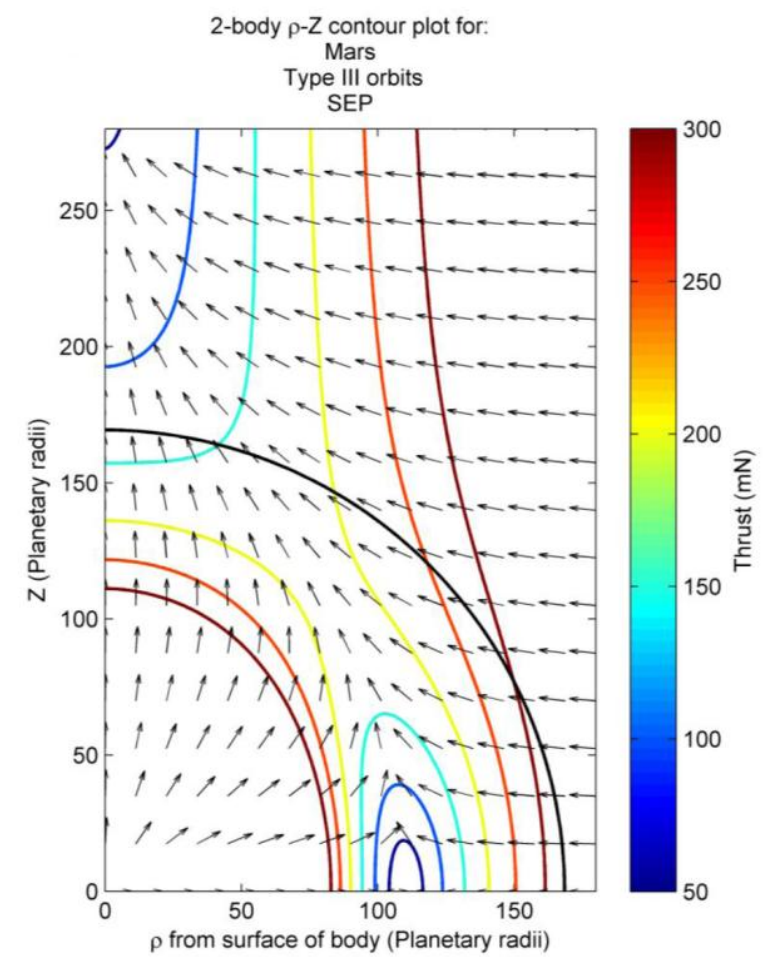

Fig. 8: Contours of constant thrust for Type III orbits about Mars using 1000kg SEP spacecraft with 300mN of thrust, synchronized with a Keplerian orbit of radius $\rho=110$ Mars radii. The thick black line again represents the approximate sphere of influence boundary of Mars. From Ref. [43].

\subsubsection{Two-body displaced orbit stability}

By adding a perturbation $\sigma$ such that $r \rightarrow \tilde{r}+\sigma$ (where $\tilde{r}=(\tilde{\rho}, \tilde{\varphi}, \tilde{z})$ corresponds to the nominal displaced nonKeplerian orbit solution, with the thrust-induced acceleration fixed in both magnitude and direction), and linearizing the equations of motion in the rotating reference frame, a variational equation can be obtained. As was pointed out in Ref. [40], the local stability characteristics of the three types of orbit can then be obtained through the characteristic polynomial of this variational equation, with linear stability corresponding to nonpositive roots - which requires that both the coefficients and the discriminant of the quadratic of the variational equation be positive. It can be shown that the discriminant for all three two-body orbit types is positive. It was also noted that, because the equations of motion have been linearized, the analysis only provides necessary conditions for stability and sufficient conditions for instability. However for Type I orbits the conditions agree with the necessary and sufficient condition obtained by Dankowicz [25] using a fully non-linear analysis in KS variables and, further, numerical experiments for Type II and III orbits suggest that these conditions for stability are indeed sufficient [40].

For Type I orbits, the necessary but not sufficient stability condition is satisfied when [40]

$$
\rho>2 \sqrt{2 z}
$$


meaning that stable and unstable families of Type I orbits exist, partitioned by the cone defined by Equation (12), with examples of such orbits being shown in Figure 9, in non-dimensional units. As discussed in the previous paragraph this agrees with the non-linear analysis of Dankowicz and thus provides both a necessary and sufficient condition for stability.

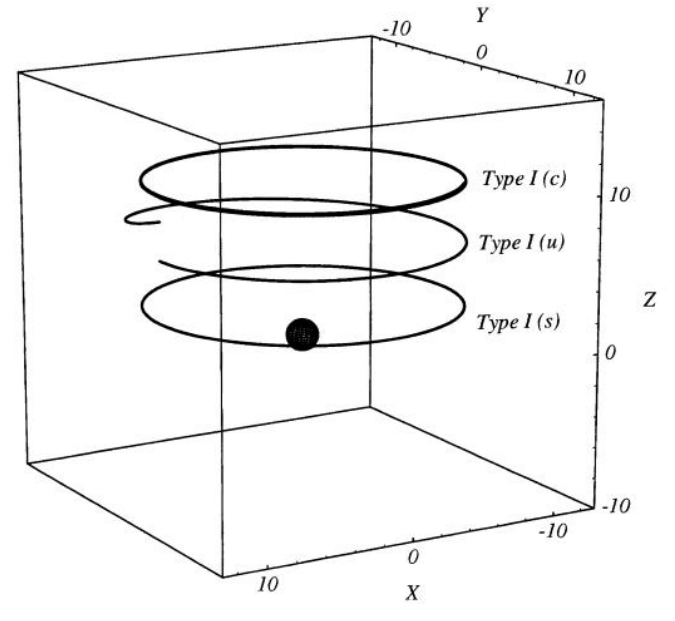

Fig. 9: Type I orbits: stable (s) $\left(\rho_{0}=10, z_{0}=2\right)$, unstable (u) $\left(\rho_{0}=10, z_{0}=6\right)$, and controlled (c) $\left(\rho_{0}=\right.$ $\left.10, z_{0}=10\right)$. From Ref. [41].

For Type II orbits the necessary but not sufficient condition for stability is given by [40, 41]

$$
\rho>2.264 \mathrm{z}
$$

which again defines a cone separating the stable and unstable orbits, with Figure 10 displaying some examples of both.

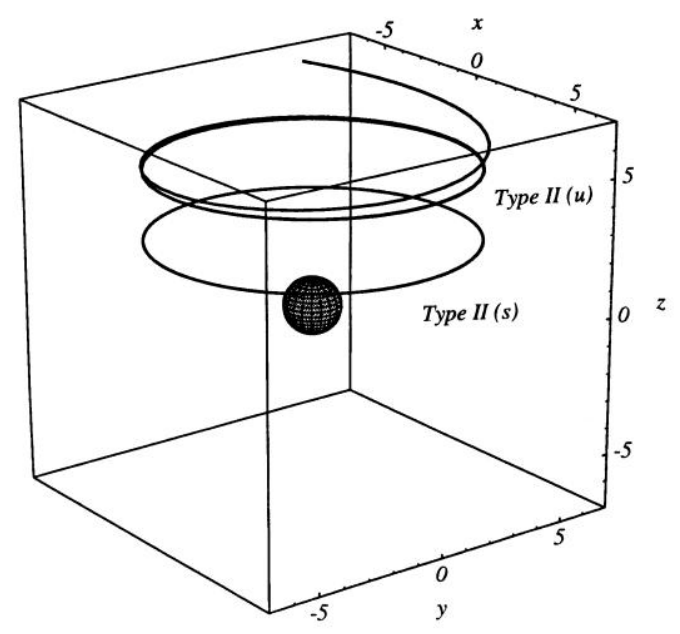

Fig. 10: Stable Type II (s) and unstable Type II (u) orbits. From Ref. [40]. 
Type III orbit stability considerations generally require a numerical solution of the stability criteria - however, for particular classes of orbits, a closed solution can be obtained. Writing the required value of $\omega_{0}$ as

$$
\omega_{0}=\sqrt{\frac{G M}{r_{0}^{3}}}
$$

with $r_{0}$ as some fixed orbit radius, then defines two coefficients and thus two stability conditions. For the first condition to be strictly positive it is necessary that

$$
r>\left(\frac{1}{3}\right)^{1 / 3} r_{0}
$$

The condition for the second coefficient to be strictly positive in general requires a numerical solution, but for highly non-Keplerian Type III orbits that are not displaced (i.e. simply lie in the $z=0$ plane), the requirement is

$$
\rho>\left(\frac{2}{3}\right)^{1 / 3} r_{0}
$$

which is a stricter condition for stability than Equation (15).

Note that more information on the stability of non-Keplerian orbits can be found in Ref. [44], which includes a plot of the stable and unstable regions of the $\rho-z$ plane for Sun-centred non-Keplerian orbits. More recently, Xu \& Xu [45] carried out a much more detailed and mathematically rigorous analysis of the nonlinear dynamical analysis and control of displaced solar sail orbits above a planet, showing that there are two equilibria - one hyperbolic (saddle) and one elliptic (centre) - as well as a saddle-node bifurcation point for the system. The authors also determined the necessary and sufficiency conditions of stability for the motion near the equilibria in a restricted two-body formulation.

\subsubsection{Two-body displaced orbit control}

Ref. [41] investigated linear orbit control of two-body displaced NKO's as a means of stabilizing the unstable families of orbits, determining the conditions for non-controllability for pitch and acceleration control. It was also shown that Type I orbits are always controllable provided that $\rho \neq 0$, and that other families of orbits are also controllable in general. 
Further examination of the control of solar sails in two-body problems is found in Refs. [46] and [47]. In the former it was demonstrated that the unstable subset of displaced solar sail orbits are in general controllable using state feedback to the sail elevation angle alone, and a passive control scheme that renders this unstable subset linearly stable was presented. Such a control scheme, which is relatively simple and requires only that the sail attitude is kept fixed relative to the Sun, may be an attractive means of orbit stabilization. In the latter a two-body stability condition for the orbits is derived using both a linear and non-linear analysis, and a Jacobi-type integral to identify zerovelocity surfaces that bound the orbital motion. A closed-form solution was derived using parabolic coordinates. A linear controller was designed to prevent escape from the highly non-Keplerian orbit, by controlling the solar sail acceleration either via sail area or sail pitch angle control. Ref. [45] also discusses control of 2-body displaced orbits in some detail.

In other work, Gong, Baoyin \& $\mathrm{Li}$ [48] considered the relative orbit design and control methods for solar sail formation flying around displaced solar orbits, discussing two types of orbit control. One method uses only position feedback to control the formation, in which the relative orbit can be controlled to be an ellipse, with the period of relative motion and direction of relative motion plane able to be designed as mission requirements. The other method uses the full state feedback to control the formation, in which the relative orbit can be designed arbitrarily, with two control strategies (Linear Quadratic Regulation and Input Feedback Linearization) discussed for this method.

\subsubsection{Connections between highly non-Keplerian two-body orbits}

For both the heliocentric and planet-centred displaced orbits discussed above an interesting extension to these families of orbits was presented by McInnes \& Simmons [18, 22], which showed that displaced orbits can be patched together, or to Keplerian orbits. By identifying conditions for continuity of energy and orbital angular momentum across orbits, complex trajectories can be formed.

McInnes outlined more detail on patched orbits in general in Ref. [41], patching non-Keplerian orbits to Keplerian orbits by nulling the spacecraft thrust. Since the spacecraft thrust vector is always normal to the velocity vector (so that the total energy $E$ is constant) for a non-Keplerian orbit, then since $E=-\mu / 2 a_{S}$, the semi-major axis $a_{S}$ of the Keplerian orbit obtained by nulling the spacecraft acceleration can be found. The patch point corresponds to the apocentre of the Keplerian orbit and thus an expression for the eccentricity can also be obtained.

Non-Keplerian orbits can also be patched to each other by considering the conditions for velocity continuity and conservation of energy and orbit angular momentum, which allows complex new families of orbits to be generated. Examples of both kinds of patched orbit are shown in Figure 11. 


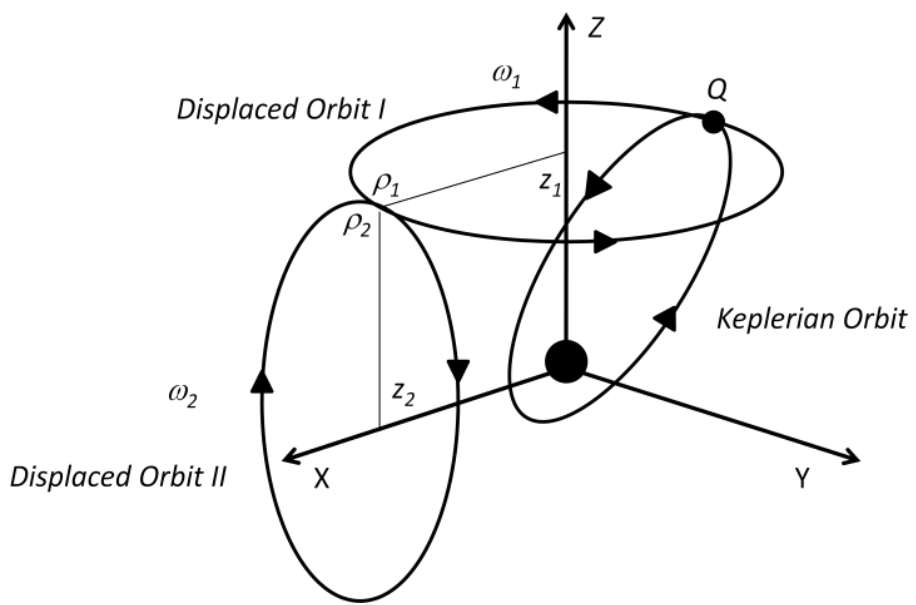

\section{Fig. 11: Schematic of patched orbits. Displaced Orbit $I$ is a reference non-Keplerian orbit, with Displaced Orbit II and Keplerian Orbit being orbits patched to this reference orbit. $Q$ is the patch point for the Keplerian orbit. From Ref. [40].}

Other patched two-body orbits include those outlined by Bookless \& McInnes [47], who found orbits that are mapped to the surface of bounding parabolas, and wind onto displaced non-Keplerian orbits. In principle, these can be used to insert a solar sail into a non-Keplerian orbit.

\section{The Three-Body Case}

The consideration of the gravitational interaction of three celestial bodies has a significant and lengthy history in the literature, with the first studies being traced back to Newton in the $17^{\text {th }}$ Century, who considered the motion of three massive bodies subject to their mutually perturbing gravitational attractions. In the three-body problem, however, as stated previously there is no closed form analytical solution for the equations governing the motion of the three bodies (unlike for the two-body case) and thus, in order to make the problem tractable, some simplifying assumptions need to be made. Euler detailed the "restricted three-body problem" by considering one of the bodies as having negligible mass with respect to the other two bodies, thus not affecting their motion as they orbit their common centre of mass in standard Keplerian two-body fashion. The "circular reduced three-body problem" (CRTBP) is simply a further reduction in complexity by requiring that the two massive bodies orbit the centre of mass in purely circular orbits. In 1772 Lagrange determined equilibrium solutions of the CRTBP [49], identifying the five (now well-known) positions where the combined gravitational pull of the two massive bodies provides precisely the centripetal force required to rotate with them, or, put in another way, at these five points a third body of negligible mass remains fixed, relative to a frame of reference that rotates with the primaries.

It therefore stands to reason that, in the same way continuous low-thrust acceleration displaced a spacecraft to an artificial equilibrium point away from a body in the two-body problem, propulsive thrust will do the same about any of these five Lagrange points. 
A specific subset of artificial equilibrium points is that of those located on the line joining the two primary masses, i.e. exactly on the collinear plane, with such points often being referred to generally as displaced, or artificial, Lagrange points. Such points by definition only refer to artificial $\mathrm{L}_{1}, \mathrm{~L}_{2}$ and $\mathrm{L}_{3}$ points - although it is of course possible to displace the $\mathrm{L}_{4}$ and $\mathrm{L}_{5}$ points in the collinear plane as well, as discussed by, for example, Schuerman [31], who first elaborated on the displacement of $\mathrm{L}_{4} / \mathrm{L}_{5}$ and showed that they move sunward, forming an arc, and Murray [33], who showed that the displacement of these point is tangential to, rather than on, the line joining the primary mass and the classical $\mathrm{L}_{4} / \mathrm{L}_{5}$ point. For the purposes of attempting to standardize the notation, artificial equilibrium points (AEPs) are defined to be the general case of a point displaced away from a natural Lagrange point in any direction and/or plane, whereas displaced Lagrange points remain in the collinear plane (with the above caveat that the references in the literature may only be referring specifically to the $\mathrm{L}_{1} / \mathrm{L}_{2} / \mathrm{L}_{3}$ points in some cases).

A few authors have considered periodic orbits about such displaced artificial Lagrange points. These periodic orbits are analogous to the classical "halo" orbits. Further, a subset of this work is that of the small body of research on periodic orbits about AEPs high above the ecliptic plane. Such work is considered in more depth in Section 3.4. First, a model for determining the locations of artificial equilibrium points in the three-body model is provided, and the effects of different propulsion systems considered.

\subsection{Model}

As discussed earlier it was Dusek [7] who initially considered the case of libration points with continuous control acceleration, showing that artificial equilibrium points can be generated by constant low-thrust radial forces. However it was McInnes et al. [41] who determined the conditions for circular displaced highly non-Keplerian orbits, by considering the dynamics of a spacecraft of mass $m$ in a reference frame $\boldsymbol{R}(x, y, z)$ in which the primary masses $m_{1}$ and $m_{2}$ are fixed (as shown in Fig. 12, where the $x$-axis points between the primary masses, the $z$-axis denotes the axis of rotation and the $y$-axis is orthogonal to both).

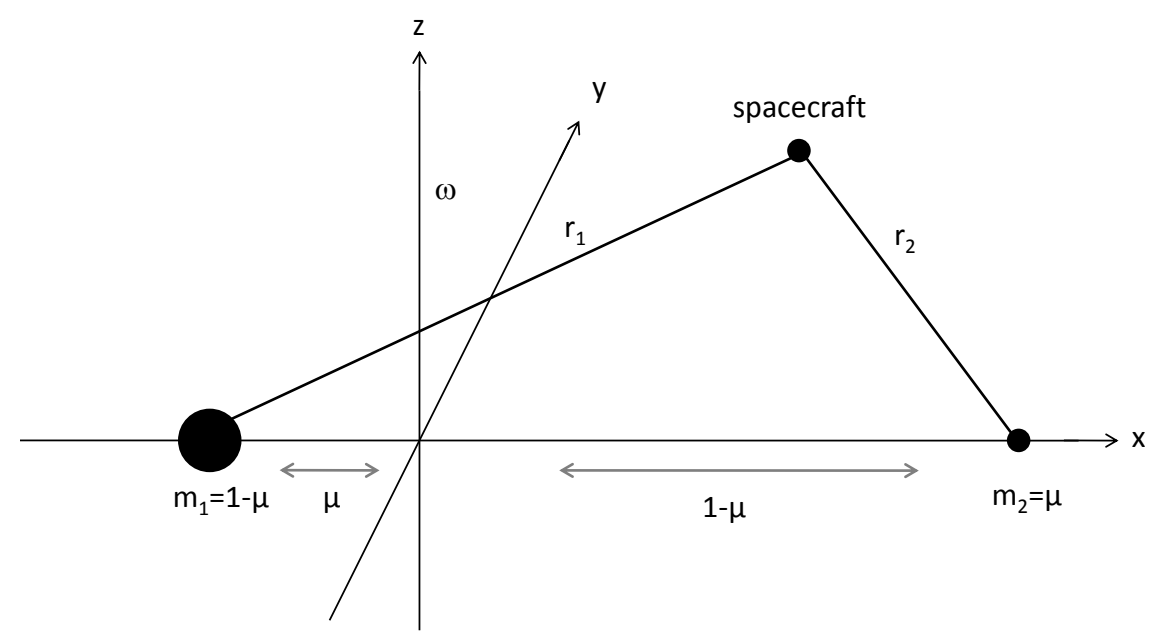

Fig. 12: The rotating coordinate frame and the spacecraft position therein for the restricted three-body problem. 
This reference frame rotates at constant angular velocity $\boldsymbol{\omega}$ relative to an inertial frame $\boldsymbol{I}(X, Y, Z)$. With such a system the equations of motion of the spacecraft are given by

$$
\ddot{\boldsymbol{r}}+2 \boldsymbol{\omega} \times \dot{\boldsymbol{r}}+\nabla V=\boldsymbol{a}
$$

where $\boldsymbol{r}$ is the position vector of the spacecraft in the rotating frame, dots denote differentiation with respect to time $t$, and $V$ and $\boldsymbol{a}$ are the augmented potential and the continuous and constant acceleration due to the propulsion system respectively. The augmented potential is given by,

$$
V=-\left[\left(\frac{1-\mu}{\left\|r_{1}\right\|}+\frac{\mu}{\left\|r_{2}\right\|}\right)\right]-\frac{1}{2}\|\boldsymbol{\omega} \times \boldsymbol{r}\|^{2}
$$

in units where the gravitational constant $G=1$, the system has total unit mass, the position vectors $\boldsymbol{r}_{1}$ and $\boldsymbol{r}_{2}$ of the spacecraft with respect to the primary masses $m_{1}$ and $m_{2}$ are given by $\boldsymbol{r}_{1}=(x+\mu, y, z)^{T}$ and $\boldsymbol{r}_{2}=((x-$ $(1-\mu)), y, z)^{T}$ respectively, and where $\mu$ is the reduced mass,

$$
\mu=\frac{m_{1}}{m_{1}+m_{2}}
$$

The continuous and constant acceleration from the low-thrust system is given by

$$
\boldsymbol{a}=\left[\frac{T}{m}\right] \boldsymbol{n},
$$

where $\boldsymbol{n}$ is the direction of the thrust $T$ (where this equation makes no assumptions about the propulsion system used, so for e.g. a solar sail Equation (20) must be modified accordingly). By setting $\ddot{\boldsymbol{r}}=0$ and $\dot{\boldsymbol{r}}=0$, i.e. assuming equilibrium conditions in the rotating frame, then the equation $\nabla V=\boldsymbol{a}$ defines a surface of equilibrium. With such a formulation then a spacecraft occupying an artificial equilibrium point tracks out a periodic orbit in the inertial reference frame, with the period being defined by that of the body which the spacecraft is in equilibrium with.

Further, the required thrust vector orientation for an equilibrium solution is then given by,

$$
\boldsymbol{n}=\frac{\nabla V}{\|\nabla V\|}
$$


and the magnitude of the thrust vector, $\|\boldsymbol{a}\|$, is given by,

$$
\|\boldsymbol{a}\|=\|\nabla V\|
$$

As can be seen from Eq. (22), by definition, for such equilibrium solutions the value of the parameter $\lambda$ is exactly equal to 1. Further, the equation for the magnitude of the acceleration vector $\|\boldsymbol{a}\|$ defines an implicit function in the rotating coordinates. As an implicit function can be expressed in the form $f(x, y, z)=0$ it defines a threedimensional equithrust surface which can be plotted (an example of which is shown in Section 3.2). Precisely which equilibrium surfaces are accessible depends on the mass ratio of the two massive bodies, the amount of thrust available to the spacecraft (of given mass) in question, and the orientation in which the propulsion system is able to deliver said thrust.

As might thus be expected, the work in studying artificial equilibrium points has evolved to consider multiple forms of propulsion. As discussed previously, much of the original work was initially considered in the framework of solar sailing [34]. However, due to the orientation constrained nature of solar sail propulsion (e.g. not being able to thrst towards the Sun), highly non-Keplerian orbits enabled via solar sails can be thought of as being a subset of the more general set of NKOs generated by non-orientation constrained low thrust propulsion systems, such as solar electric propulsion.

Thus this section proceeds by discussing the case of solar electric propulsion, before then moving on to the case of solar sails. Unlike the wide-ranging historical overview outlined in Section 1, here much more recent and much more focused work is discussed primarily, in order to give a feel for the subject as it stands currently, as opposed to how it has developed historically.

\subsection{Highly non-Keplerian orbits with solar electric propulsion}

Morimoto et al. [50] have considered the problem of artificial equilibrium points in a very generalized way, analyzing arbitrary AEPs in the CRTBP for the general mass-ratio range with an idealized continuous low thrust, in order to allow for more flexible and generic mission designs than considering the more narrowly-focused cases of specific libration points, common planetary mass ratios and/or current-term low thrust acceleration ranges. The magnitude and direction of the required acceleration for an AEP was considered in three-dimensional space, and additionally a linear stability analysis was carried out. It was shown that the acceleration to enable an AEP required the presence of small close, natural equilibrium points, independent of the mass ratio of the system, and further that for small mass ratio systems there are stable regions of AEPs with very small control acceleration. The identification of regions of stable AEP's is important, since the solar sail does not admit such regions [34]. Additionally, in other work the same authors focus on resonant periodic orbits at linear order existing on the line connecting two primary bodies with a continuous low-thrust propulsion system [51]. 
In contrast to the work of Morimoto et al., more recently McKay et al. [43] carried out a more specific study of artificial equilibrium points and surfaces throughout the solar system, assuming a spacecraft of mass $1000 \mathrm{~kg}$ equipped with an SEP thruster with a maximum thrust of $300 \mathrm{mN}$ and a specific impulse $\left(\mathrm{I}_{\mathrm{SP}}\right)$ of 4500 seconds. These assumptions allowed for a consideration of opportunities based on current or near-term SEP technology such as the Qinetiq T6 thruster, which will theoretically provide a thrust of up to $230 \mathrm{mN}$ at an $\mathrm{I}_{\mathrm{SP}}$ of above 4500 seconds for the BepiColombo mission [52], although such capabilities still have to be demonstrated within the context of any space mission.

The left-hand panel of Figure 13 provides an example of the artificial equilibrium points about Mercury projected onto planes parallel to and perpendicular to the orbital plane respectively, with the right-hand panel showing one of the equithrust surfaces about the same body. Anywhere on the equithrust surface, a spacecraft with the required thrust, oriented in the direction needed, will exist in equilibrium with the body in question.

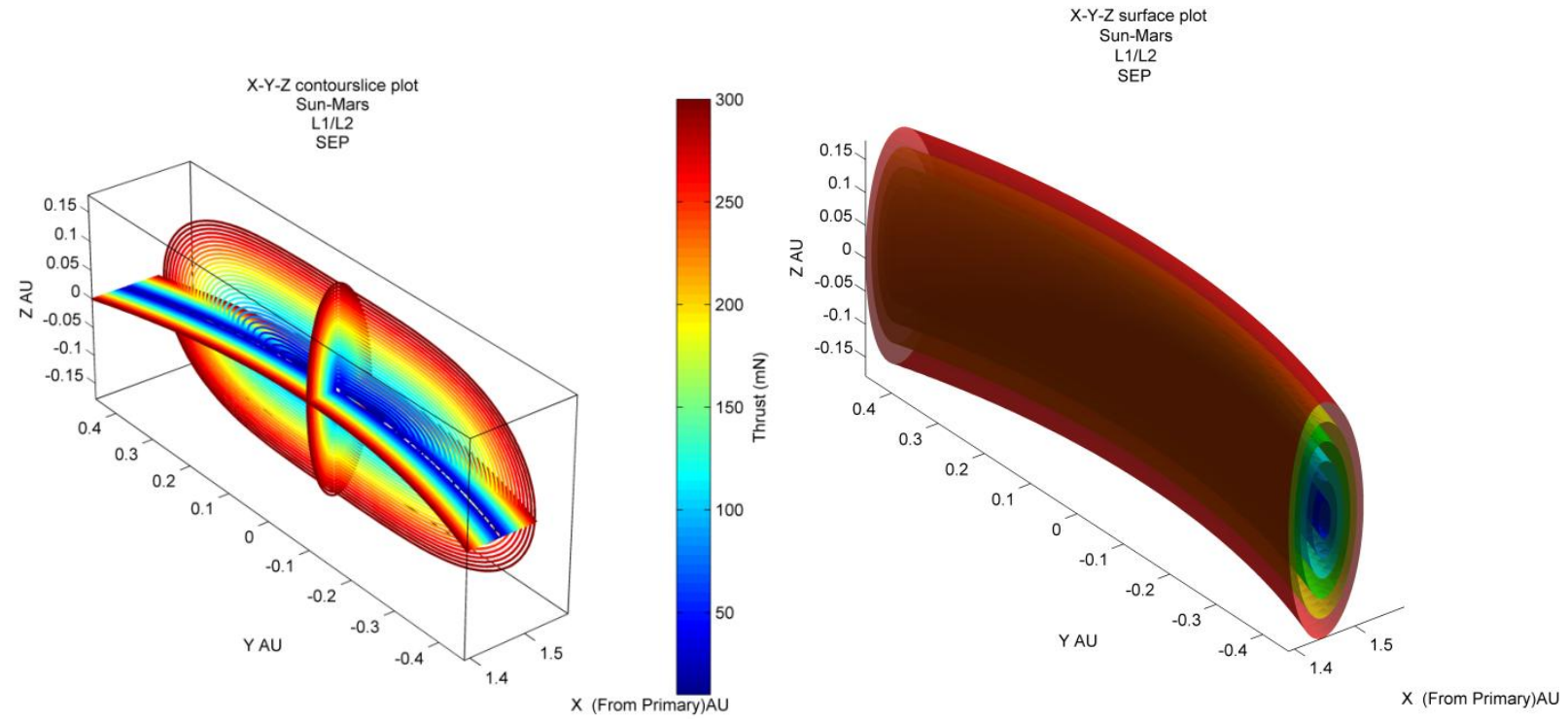

Fig. 13: Artificial equilibrium points depicted by equithrust contours projected onto the planes perpendicular to and parallel to the orbital plane (left), and the 50 (dark blue), 100, 150, 200, 250 and $300 \mathrm{mN}$ (red) equithrust surfaces (right) for the Sun-Mars $L_{1} / L_{2}$ system.

As one moves far away from the second body in the three-body problem, the contours for the two- and three-body problem become identical, assuming that the orbit period is still fixed to that of the secondary body. This simply means that the orbits far away from the secondary body in a three-body problem essentially reduce to that of a twobody Type III problem. 


\subsection{Highly non-Keplerian orbits with solar sails}

The principle of solar sailing relies on the radiation pressure and subsequent momentum transfer of photons reflecting off a large mirror. With a sufficiently large sail mirror area, then a substantial thrust can be generated. The beauty of solar sailing is thus in the lack of reaction mass required to propel the spacecraft, with the continual and significant radiation pressure from the Sun being able to accelerate a solar sail to a considerable speed over a period of time. Equally, this continuous thrust can be used to perform highly non-Keplerian orbits.

As discussed previously, the initial studies of artificial three-body equilibria for the solar sail were made in Ref. [34], where regions of existence of artificial Lagrange points were identified, with the required sail orientation and the required sail loading in the Sun-Earth restricted three-body system determined. The origin of the coordinate system is taken to be the centre of mass of the two primaries, as previously shown in Figure 12, with the position vector of the solar sail with respect to the two masses $m_{1}$ and $m_{2}$ is the same as given in Section 3.1. The simple form of Equation (22) for the continuous and constant acceleration of the spacecraft is instead replaced, however, by

$$
\boldsymbol{a}=\beta \frac{1-\mu}{r_{1}^{2}}\left(\hat{\boldsymbol{r}}_{1} \cdot \boldsymbol{n}_{\boldsymbol{s}}\right)^{2} \boldsymbol{n}_{\boldsymbol{s}}
$$

to instead model the acceleration of the sail due to solar radiation pressure, although it should be noted that Eq. (23) is valid only for an ideal sail (i.e. one in which the reflectivity is assumed to be perfect). Here $\boldsymbol{n}_{\boldsymbol{s}}$ is the unit normal to the sail, $\beta$ is the sail lightness number (ratio of solar radiation pressure acceleration to solar gravitational acceleration), and $\hat{\boldsymbol{r}}_{1}$ is directed along the Sun-line. The sail normal is usually given in terms of the clock and cone angles (see e.g. Refs. $[34,37]$ ), where the cone angle $\alpha$ is defined as the angle between $\boldsymbol{n}_{\boldsymbol{s}}$ and $\hat{\boldsymbol{r}}_{1}$ and the clock angle $\delta$ is defined as the angle of the projection of $\boldsymbol{n}_{\boldsymbol{s}}$ in the plane normal to $\hat{\boldsymbol{r}}_{1}$ with respect to a reference direction $\hat{\boldsymbol{r}}_{1} \times\left(\boldsymbol{\omega} \times \hat{\boldsymbol{r}}_{1}\right)$. The solar sail lightness number required for equilibrium can be determined to be [37]

$$
\beta=\frac{\mathrm{r}_{1}^{2}}{1-\mu} \frac{\nabla V \cdot \boldsymbol{n}_{\boldsymbol{s}}}{\left(\hat{\boldsymbol{r}}_{1} \cdot \boldsymbol{n}_{\boldsymbol{s}}\right)^{2}}
$$

Having determined a function for the sail lightness number required for equilibrium solutions in the rotating frame of reference, solutions of artificial equilibria can then be generated by specifying values for the lightness number and attitude, with a particular solution on a given surface being defined by the sail cone and clock angles. This was done for a variety of values of $\beta$ in Ref. [34], which assumed a perfectly-reflecting solar sail. In reality the efficiency of the sail, $\eta$, in reflecting photons is not perfect, due to the billowing of the sail film and the non-perfect optical properties of the film coating ( $\eta=1$ being perfect specular reflection). Ref. [36] considered equilibrium curves for a non-perfect sail. This is of importance because, aside from reducing the magnitude of the solar radiation pressure exerted on the sail surface, the partial reflectivity and hence finite absorption means that the radiation pressure force vector is no longer normal to the sail surface, and thus the equilibrium surfaces and accessible regions 
are slightly different, as compared in Figure 14 in terms of the sail loading parameter determined from the relation $\beta=\sigma / \sigma_{*}$ (where $\sigma_{*}$ is the critical sail loading, $\sigma_{*}=1.53 \mathrm{gm}^{-2}$ ). The region in which solutions exist is bounded by the equation $\hat{\boldsymbol{r}}_{1} \cdot \boldsymbol{n}_{\boldsymbol{s}}=0$.
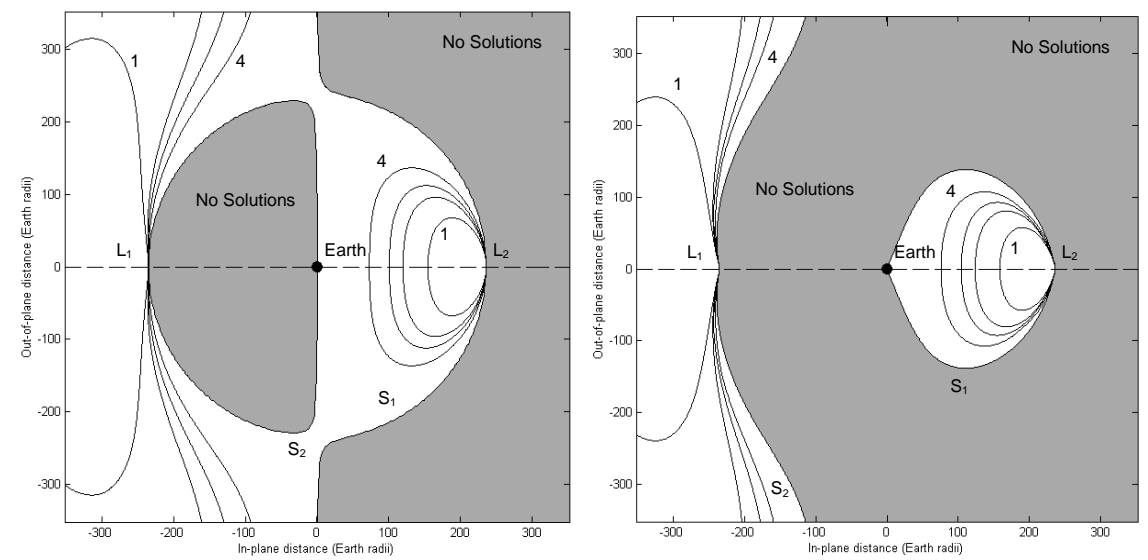

Fig. 14: Comparison of contours of sail loading in the x-z plane with sail efficiency $\eta=1$ (left) and $\eta=0.9$ (right), where the contours are, in $\mathrm{gm}^{-2}:[1] 30$ [2] 15 [3] 10 [4] 5 .

To make the solar sail and SEP equilibrium contours more instantly comparable McKay et al. considered equivalent thrust-to-mass ratios for the two systems [43], assuming a 1000kg SEP spacecraft with maximum thrust capability of $300 \mathrm{mN}$, and a $100 \mathrm{~kg}$ solar sail with maximum thrust of $30 \mathrm{mN}$ - the latter being a more realistic value of mass for a solar sail, with these values being equivalent to a sail characteristic acceleration of $0.67 \mathrm{mms}^{-2}$, or equivalently, a sail loading of $13.63 \mathrm{gm}^{-2}$. Such values are well in advance of even the most optimistic estimates of potential nearterm solar sails, but it remains a useful comparison to directly compare the SEP and solar sail propulsion against each other on equal acceleration terms about Mars. Figure 15 compares the equilibrium contours about the Sun-Mars $\mathrm{L}_{1}$ and $\mathrm{L}_{2}$ points in the $x-z$ plane for the two propulsion systems.

As can be seen, the solar sail is restricted in which equilibrium surfaces it can access compared to the nonorientation constrained case of SEP thrust, with the filled blue regions of the right-hand panel of Figure 15 representing the forbidden regions where, as the arrows indicate, the spacecraft needs to be thrusting towards the Sun in order to be on a highly non-Keplerian orbit. Additionally, even where the solar sail equilibria do exist, they are displaced less far from the ecliptic plane, as although the sail has the same thrust-to-mass ratio as the SEP spacecraft it only exerts a component of force in the direction required, unlike the SEP case where the total thrust can be directed in whichever direction is required. 

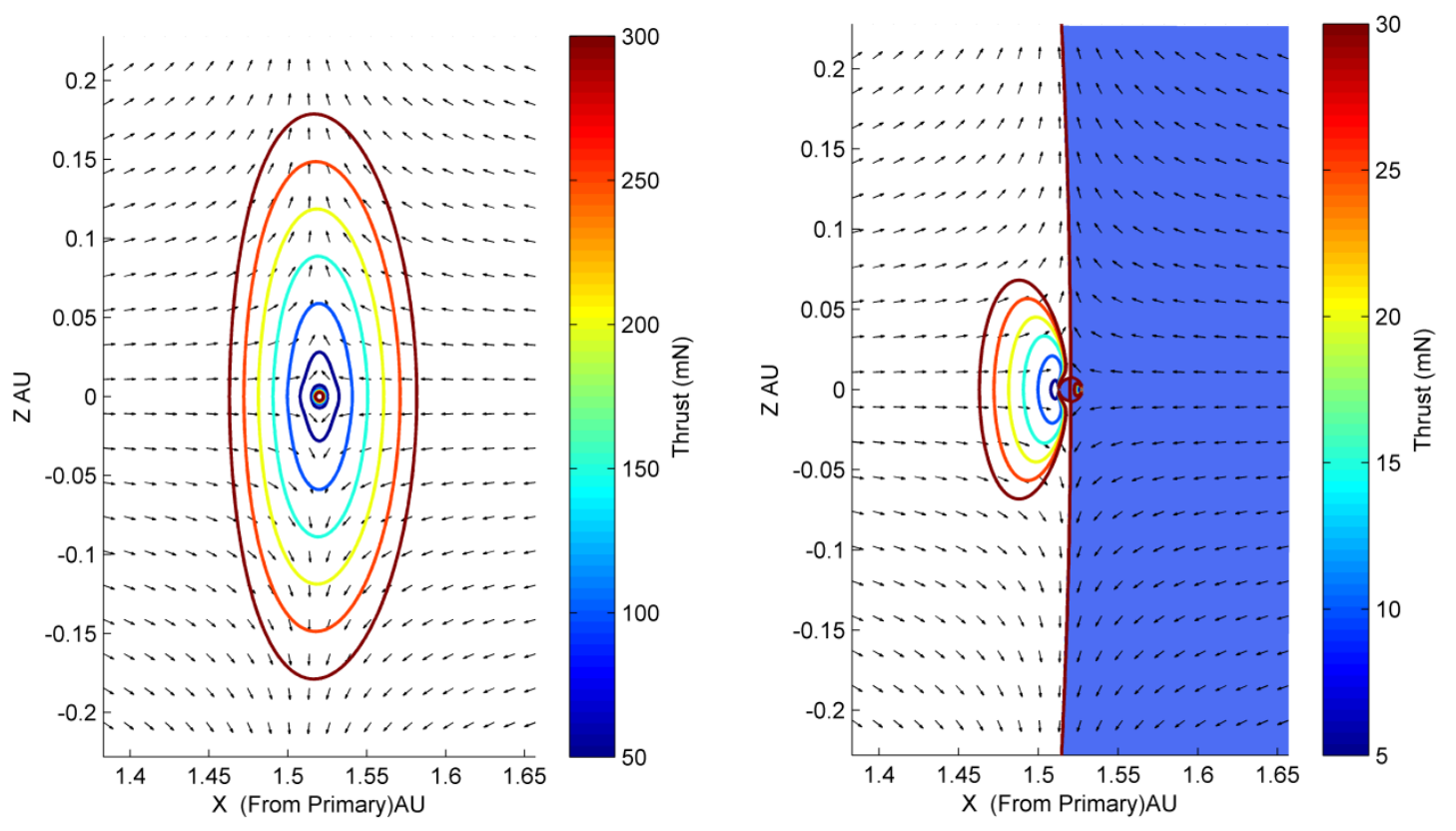

Fig. 15: Non-Keplerian orbit equithrust contours projected onto the plane perpendicular to the Ecliptic plane for the Sun-Mars-spacecraft 3-body system for SEP spacecraft (left) and solar sail (right). From Ref. [43].

Details on the linear stability properties of solar sails in the CRTBP are found in Ref. [34], where it was shown that the equilibrium surfaces are unstable and that Lyapunov stability is only possible for the particular solutions where the sail is oriented along the sun line. Thus a simple control scheme using proportional and derivative feedback to the sail attitude was devised in this paper, in order to ensure asymptotic stability. McInnes [53] and Lawrence \& Piggott [54] also presented some preliminary investigations into variations of a solar sail's orientation, the natural mechanism of control for a sail, to determine the robustness of such a mechanism. In the latter case the authors considered a $10,000 \mathrm{~m}^{2}$ sail and showed that it is controllable on the station-keeping manifold in the case of a circular Earth orbit about the Sun, and further, indicated whether orbit injection errors (relative to the desired station) are correctable for a given mission profile and quantified tracking error correctability along non-equilibrium trajectories, by using a controllability Gramian approach. In other work Bookless \& McInnes [47] treated the threebody problem as a perturbed two-body model and outlined an optimal controller designed to select control gains that minimize the sail area or pitch-angle variation required to control displaced non-Keplerian orbits. Further, they demonstrated how a non-Keplerian orbit with a robust controller could enable long-duration science missions within the geomagnetic tail.

More recently, Bombardelli and Peláez [55] have carried out an analysis of the stability properties of minimumcontrol artificial equilibrium points in the CRTBP, showing that when the masses of the two primaries are of different orders of magnitude, minimum-control equilibrium is obtained when the spacecraft is almost co-orbiting with the second primary - as long as their mutual distance is not too small. Ref. [55] also showed that stability is found when the distance from the second primary exceeds a minimum value (which is a simple function of the mass 
ratio of the two primaries and their separation) and, further, that the concept could be applied to demonstrate observatories co-orbiting with the Earth, Mars, and Venus, stabilized by means of a solar sail.

\subsection{Hybrid SEP/sail}

As has been noted in the previous two sections, both SEP and solar sail low thrust propulsion systems have their own advantages and disadvantages. Solar sailing has the advantage of an effectively infinite specific impulse, i.e. requires no propellant/fuel, and thus can maintain continuous low thrust indefinitely (in theory, at least - in practice, long-term degradation of the optical surface may reduce the efficiency of the sail $[56,57])$. However with SEP one is free to orient the thrust in any direction, allowing access to artificial equilibria that a solar sail may be forbidden from with its inherent inability to thrust towards the Sun. Thus in theory there is a strong case for studying a device that would combine the best features of both systems, to obtain a hybrid sail: indeed, it has recently been suggested that such an approach may, in recognition of the high Advancement Degree of Difficulty of solar sailing, be the best means of advancing solar sail technology [58]. Such an idea has been shown to be potentially important, given the challenges of performing complex missions - see, e.g. Leipold \& Götz [59] and Mengali \& Quarta [60], with the latter showing that hybrid sails have the attractive feature of reducing mission times for heliocentric transfers when compared to both the equivalent pure sail and pure SEP trajectories. Recently Baig \& McInnes [61], Simo \& McInnes [62] and Ceriotti \& McInnes [63] have considered the case of displaced highly non-Keplerian orbits for the specific case of a hybrid sail.

Generally consideration of a hybrid sail consists of an SEP thruster attached to the centre of the solar sail. Ref. [61] considered a partially reflecting hybrid sail model, adapted from that of Ref. [59], as shown in Figure 16, where $\boldsymbol{n}_{\boldsymbol{s}}$ and $\boldsymbol{t}_{\boldsymbol{s}}$ are the unit vectors normal to and transverse to the hybrid sail surface, respectively, $\hat{\boldsymbol{r}}_{1}$ is the direction of incident photons and the unit vector $\mathbf{s}$ defines the direction of specularly-reflected photons. Vector $\boldsymbol{m}$ is the direction of the solar radiation pressure force on the hybrid sail, the offset angle between $\boldsymbol{m}$ and $\boldsymbol{n}$ is usually called the centre line angle $(\varphi), \theta$ is the cone angle, and angle $\alpha=\theta+\varphi$. With such a model the total thrust of the hybrid sail due to both solar radiation pressure and solar electric propulsion is given by [61]

$$
\mathbf{F}_{T o t}=\mathbf{F}_{S E P}+\mathbf{F}_{\text {Sail }}=F_{n} \boldsymbol{n}_{\boldsymbol{s}}+F_{t} \boldsymbol{t}_{\boldsymbol{s}}+\mathrm{T} \boldsymbol{u}
$$

where $F_{n}$ and $F_{t}$ denote the forces normal to and transverse to the sail respectively (expressions for which can be derived via consideration of the projected area of the hybrid sail in the direction of the incident photons), and $T$ denotes the thrust from the SEP, where the unit vector $\boldsymbol{u}$ denotes the thrust direction. 


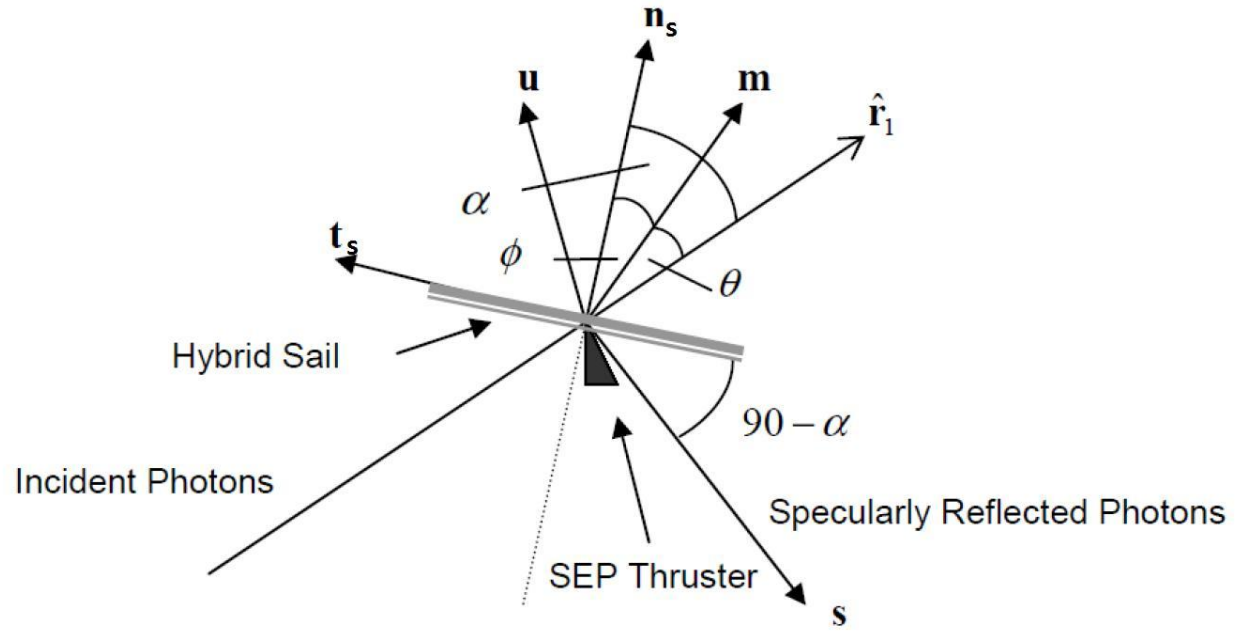

Fig. 16: Specularly reflecting hybrid sail solar radiation pressure force model. From Ref. [61].

Ref. [61] notes that, for a hybrid sail, the desired acceleration vector to keep the hybrid sail at an artificial equilibrium point is achieved by the sum of the solar radiation pressure and SEP acceleration vectors, and compared the families of thrust acceleration vector contours near the Earth in the $x-z$ plane for the case of pure SEP and a hybrid sail, as shown in Figure 17 for a solar sail with dimensionless lightness number $\beta_{0}=0.05$, where $\beta_{0} \equiv$ $\sigma_{*} /\left(m_{0} / A_{T}\right)$. Here $\sigma_{*}$ is the critical sail loading parameter $\left(1.53 \times 10^{-3} \mathrm{~kg} \mathrm{~m}^{-2}\right), m_{0}$ is the initial mass of the hybrid sail, and $A_{T}$ is the total area of the hybrid sail (i.e. the sail area plus the thin film area).

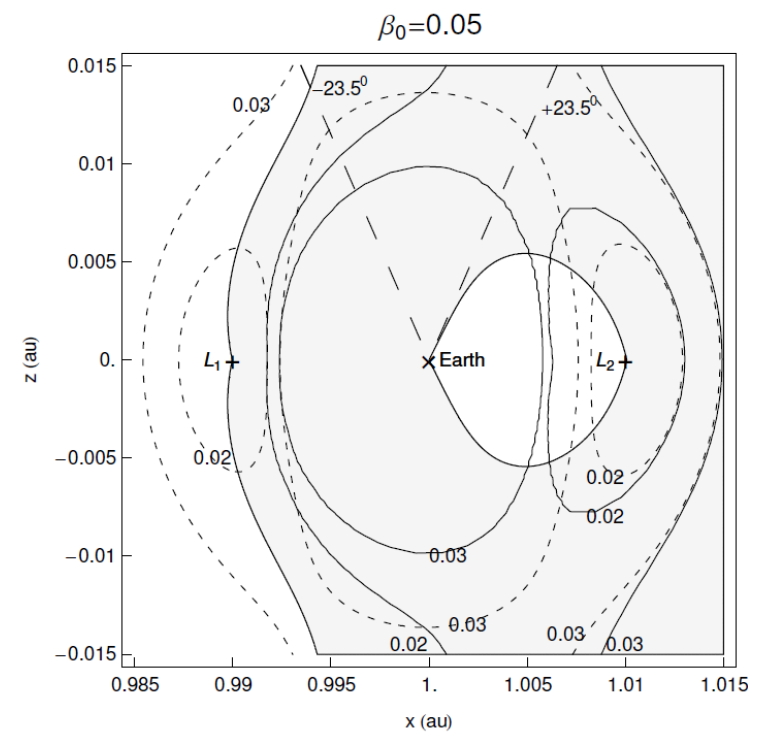

Fig. 17: Thrust acceleration contours in the $x-z$ plane. Non-dimensional acceleration values $0.02,0.03$ are shown by dotted lines for a pure SEP system and solid lines for the hybrid sail. The hybrid sail has an effective dimensionless lightness number of 0.05 . From Ref. [61].

The shaded region represents the region where a pure sail cannot be placed. Ref. [61] showed that the hybrid sail has benefits over both pure sail and pure SEP systems. In the former case, this is because the hybrid sail can access 
regions the pure sail cannot, due to the addition of the SEP thruster which can be oriented in any direction. In the latter, a larger volume of space is available for artificial equilibrium solutions around $\mathrm{L}_{2}$ with the hybrid sail. However it is clear that there are locations beyond $\mathrm{L}_{1}$ where the hybrid sail is not as effective as a pure SEP system, because the hybrid sail cannot use the solar radiation pressure effectively there and the small SEP system provides insufficient force to compensate. However, the hybrid sail shows benefits over the pure solar sail, for example in artificial equilibria above the poles of the Earth. The dashed line in Figure 17 shows the Earth's polar axis at the winter and summer solstice - it can be seen that the hybrid sail contours are closer to the Earth than the pure SEP contours.

\subsection{Periodic orbits about artificial equilibrium points}

As was discussed at the start of Section 3, analysis on the collinear libration points in the restricted three-body problem has revealed an additional class of three-dimensional, periodic "halo" orbits. A spacecraft in a halo orbit does not technically orbit the Lagrange point itself (which is just an equilibrium point with no mass), but travels in a closed, repeating path near the Lagrange point - specifically, the $\mathrm{L}_{1}, \mathrm{~L}_{2}$ and $\mathrm{L}_{3}$ points. Halo orbits are the result of a complicated interaction between the gravitational pull of the two primary bodies and the centripetal acceleration on a spacecraft. It was Robert Farquhar who coined the term "halo" for such orbits in his PhD thesis of 1968 [64], having initiated an analytical investigation into such precisely-periodic orbits. Halo orbits are closed, periodic threedimensional orbits in the vicinity of the $\mathrm{L}_{1} / \mathrm{L}_{2} / \mathrm{L}_{3}$ points of the three-body system, being the natural motion of a satellite around a collinear libration point in a three-body system. Along with Kamel, Farquhar then developed analytical solutions for quasi-periodic orbits about the $\mathrm{L}_{2}$ equilibrium point in the Earth-Moon system using the Linstedt-Poincaré method [65], noting that a communications satellite in such an orbit has the advantage of continuous contact with both the Earth and the far side of the Moon - such orbits thus being permanently visible from Earth and suited to the Apollo missions of the era. Breakwell \& Brown [66] numerically extended this work, and in doing so showed that the halo orbits originating in the vicinities of both the Earth-Moon $\mathrm{L}_{1}$ and $\mathrm{L}_{2}$ points grow larger but shorter in period as they shift towards the Moon, with the orbits being fairly well-approximated by an 'almost rectilinear' analysis nearer to the Moon. Howell then furthered the use of numerical solutions and produced orbits beyond the range of validity of the analytical solutions for all three collinear libration points [67], and in doing so considered a wide range of mass ratios $\mu$ between 0 and 1 , each of which having their own stable regions of halo orbits (with the exception being for $\mathrm{L}_{1}$, which has regions of no stable points for certain values of $\mu$ the stable range decreasing with increasing $\mu$ and finally disappearing at $\mu=0.0573$ ). More recently Howell has also presented a review of periodic halo orbits and some of the associated numerical analysis therein [68].

Given this avenue of research, therefore, it is reasonable to expect that a spacecraft could also perform "halo" orbits around the continuum of artificial Lagrange points displaced away from their equivalent traditional Lagrange points by continuous low thrust propulsion, as described at the start of Section 3, thus opening up a significant wealth of new displaced halo orbits. Alan McInnes detailed halo orbits around artificial Lagrange points on the line joining the two primaries in the solar sail three-body problem in his Masters thesis [53], describing stable regions of halo orbits 
around unstable AEP's. By considering the sail attitude as directed along the Sun-sail line, several types of on-axis and controlled off-axis halo orbits were investigated. Similarly Baoyin \& McInnes [69] studied halo orbits for solar sails at artificial Sun-Earth $\mathrm{L}_{1}$ points, using a third order approximate solution, with two distinct families of such orbits arising as determined by the attitude of the sail. In the first case the sail normal was directed along the Sunsail line, and in the second case the sail normal was directed along the Sun-Earth line. The orbit period increases as the sail lightness number increases in the former case and in the latter case the orbit period increases as sail lightness number increases to around 0.01 , and then decreases as the lightness number exceeds $\sim 0.01$.

In the same way that artificial Lagrange points are simply a specific subset of AEPs on the line between the two primary masses, these artificial "halo" orbits are simply the specific subset of periodic orbits about AEPs in the plane of the two masses. One can then expand the problem to consider periodic orbits about AEPs high above/below the ecliptic plane. Technically the name "halo" no longer applies, as, viewed from one body looking along the line to the other, the orbit is displaced above or below the body and thus does not form a ring around it: however, as noted the artificial "halo" orbits are just the specific case of these periodic orbits about AEPs in the collinear plane. For more on such orbit families, see e.g. Waters \& McInnes [70], who developed families of unstable artificial periodic orbits for a solar sail about an arbitrary artificial equilibrium point high above the ecliptic plane (see Figure 18).

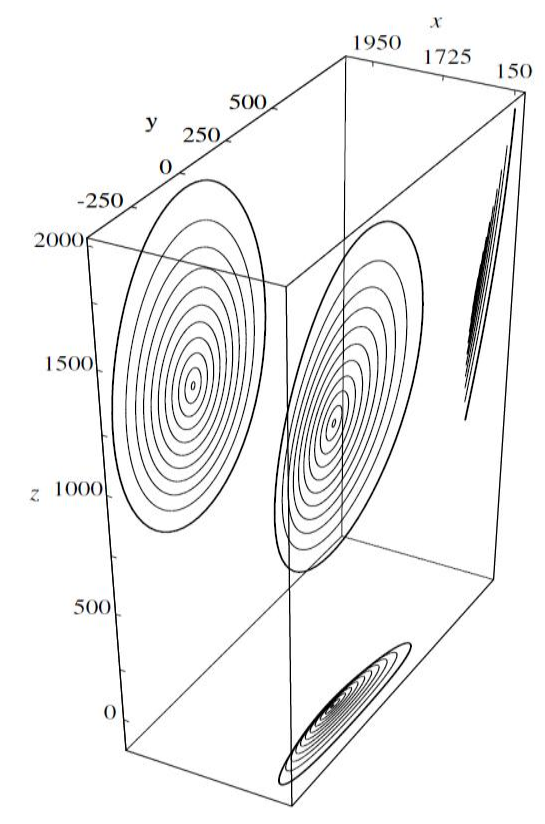

Fig. 18: Some periodic orbits about the equilibrium point $x_{e}=0.9895, z_{e}=0.0078$, with the projection of these orbits in the $x-y, y-z$ and $x-z$ planes shown. The units are given in $10^{3} \mathrm{~km}$, with the $x$ axis showing distance from the Earth. From Ref. [70].

Families of closed orbits above the ecliptic were identified using the method of Lindstedt-Poincare to find periodic approximations to the nonlinear solutions. Since the out-of-plane coordinate for the equilibrium point is non-zero for such orbits it transpires that the linear orbit solution has sufficient freedom to allow periodic orbits to be generated, 
without resorting to frequency matching techniques of classical halo orbits. The acceleration required to maintain these periodic orbits may vary over the course of the orbit period, but the time average of the acceleration will be approximately equal to that of the acceleration of the artificial equilibrium point being linearized about (provided the displacement from the AEP is small), and thus the definition for a highly non-Keplerian orbit that $\lambda$ is at least equal to 1 over the time-average of the orbit is still satisfied.

The key point of note here is that such orbits are periodic in the rotating frame, which is an extension of the orbits described previously which were only periodic in the inertial reference frame. Because of the instability of such orbits, station-keeping techniques are required to prevent the spacecraft escaping after being inserted into the orbit.

Further work by Waters \& McInnes [71] described an optimal controller to control the sail onto fixed points and periodic orbits about fixed points in the CRTBP, using solutions of the Ricatti equation, and demonstrated that a sail may be controlled onto a fixed point from quite large displacement in phase space, and also that the controller is very robust under a number of simple tests.

Bookless and McInnes [72] considered the control of Lagrange point orbits using solar sail propulsion, in order to provide station-keeping at quasi-periodic orbits around $\mathrm{L}_{1}$ and $\mathrm{L}_{2}$. Ref. [72] identified stable manifolds, which provide near-Earth insertion to a quasi-periodic trajectory around the libration point, and possible control techniques were investigated (including solar sail area variation, and solar sail pitch and yaw angle variation). Since there are points inside $\mathrm{L}_{1}$ and beyond $\mathrm{L}_{2}$ where a solar sail cannot be placed, Baig \& McInnes [73] then extended this work to consider the more general case of solar electric propulsion, in order to generate artificial halo orbits around points inaccessible to a sail.

In other relevant work Farrés and Jorba [74] studied the non-linear dynamics of a region close to a collinear equilibrium point that lies between the Earth and the Sun when a solar sail is perpendicular to the Sun-sail direction, paying special attention to the bounded motion. It was shown that for different fixed sail orientations families of planar, vertical and halo-type orbits are found. Additionally, the centre manifold was calculated around different equilibria, allowing the quasi-periodic motion around them to be described, and, further, it was illustrated that the geometry of the phase space varies with the orientation of the solar sail.

Thus, bearing in mind the discussion of Sections 2 and 3 so far, the entire family of highly non-Keplerian orbits studied to date can be summarized within a single diagram, as shown in Figure 19. On the right-hand side the three types of two-body orbits, parameterized by orbital period, are shown. The left-hand side shows the resulting artificial equilibrium points that arise from considering the three-body problem, with a subset of these being the displaced Lagrange points that are often referred to in the earlier literature (these being, as discussed previously, only those AEPs that are in the collinear plane of the two massive bodies). An extension of the AEPs is that of the periodic orbits about AEPs, as discussed initially by Waters \& McInnes [70], and, in the same way the AEPs have a 
subset of displaced Lagrange points, the artificial "halo" orbits are simply the specific subset of periodic orbits about AEPs in the plane of the two masses. At the bottom of the figure the dotted line indicates how, far from the secondary body in the three-body case, the problem simply reduces to (i.e. is equivalent to) that of a two-body Type III problem where the period is fixed to that of the secondary body.

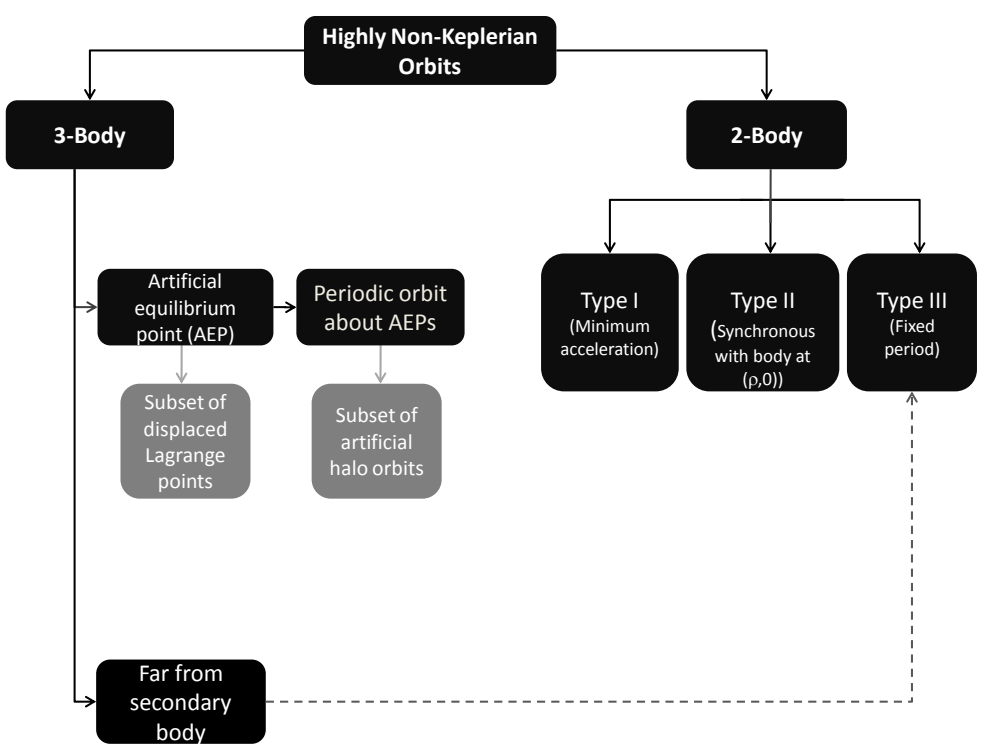

Fig. 19: Summary of displaced highly non-Keplerian orbits with continuous thrust, to date. Based on a figure from Ref. [43].

\subsection{Connections between highly non-Keplerian three-body orbits}

Invariant manifolds have been used extensively in the literature to determine low cost transfers (see, for example, the work of Belbruno, started by the application of low energy transfer orbits to the Japanese spacecraft Hiten [75] and summed up in a comprehensive book chapter on the subject [76], as well as e.g. Koon et al. [77], Gómez et al. [78], Howell \& Kakoi [79], Howell et al. [80], Pergola et al. [81]). Invariant manifolds are potentially useful in the context of solar sailing, since sail orientation can be fixed along the manifold and hence minimizes the maneuvering demands. The previous references did not consider low thrust spacecraft, however Gong et al. [82] determined the invariant manifold connection for a solar sail.

Linearizing about a fixed artificial equilibrium point determines the eigenvalue spectrum of that point, which in turn shows the structure of the invariant manifold. In the system as considered in Ref. [70] there is one stable and one unstable manifold associated with each fixed point, which characterize the flow onto and away from the fixed point. With such manifolds there are two possible scenarios for transfers: homoclinic and heteroclinic paths. 
Homoclinic paths join equilibrium points to themselves - the unstable manifold is identical to the stable manifold and hence there is a trajectory that leaves the fixed point before returning to it. Heteroclinic paths are trajectories that join two distinct equilibrium points, i.e. the unstable manifold of one point smoothly meets the stable manifold of another. They are asymptotically stable trajectories, which mean that they always converge to the target point that is, they do not oscillate around the equilibrium point.

In Ref. [70] some possible trajectories to insert a solar sail onto a large amplitude reference periodic orbit from the vicinity of the Earth were illustrated, with the stable invariant manifold - on which trajectories wind onto the period orbit - being described. The portion of the stable invariant manifold of the reference orbit that passes close to the Earth is shown in Figure 20, and the solar sail can be injected onto one of these trajectories which will naturally wind onto the periodic orbit.

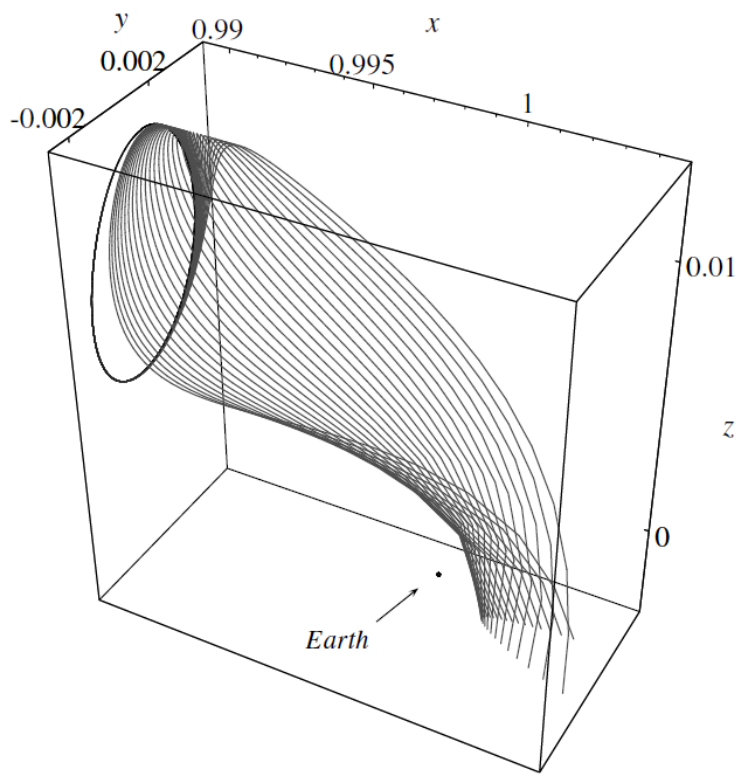

Fig. 20: Some possible trajectories for insertion into a large-amplitude periodic orbit. From Ref. [70].

The same authors found that there are equilibria admitting homoclinic paths where the stable and unstable invariant manifolds are identical, and that periodic orbits about these equilibria also admit homoclinic paths, with the entire unstable invariant manifold winding off the periodic orbit before winding back onto it in the future [83]. Such dynamics are a deep and complex field within themselves. In a separate work the same authors devised a sample solar sail mission around such invariant manifold transfers [71], which involved transfer from the Earth and between fixed points. One of the legs consisted of a transfer from a fixed point on the $\mathrm{L}_{1}$ side of Earth to a fixed point on the $\mathrm{L}_{2}$ side, using what was termed a "controlled heteroclinic transfer". With true heteroclinic paths being more difficult to find, here the unstable manifold of one fixed point simply passes close enough to another fixed point for the 
spacecraft's controller to finish the transfer. This can be done provided the solar sail is not moving too quickly as it approaches the target, which may not be the case for non-heteroclinic transfers.

In other related work, Hughes \& McInnes considered the case of trajectory optimisation for non-Keplerian orbit transfers using hybrid propulsion (see Ref. [20]). More recently, Farrés and Jorba [84] have demonstrated a control strategy to maintain a solar sail close to an unstable equilibrium point, and developed a strategy to move around the family of unstable equilibrium points, applying these strategies for a solar sail going from one region to another in the Sun-Earth planar restricted three-body problem. These techniques are based on understanding how the invariant manifolds vary as the sail orientation changes.

\subsection{Highly NKOs and other propulsion systems}

Beyond the propulsion systems as discussed in Sections 3.2-3.4, there are other more exotic methods of using low thrust propulsion to enable highly non-Keplerian orbits discussed in the literature, albeit at much earlier stages of development.

One such system is that of Pekka Janhunen's electric sail [85] which is a method of propellantless propulsion that utilises the momentum flux of the solar wind. This is achieved by an electron gun on-board the spacecraft maintaining a set of long thin wire "spokes" at high potential, to enable the wires to repel and deflect incident solar wind protons and thus provide thrust. Janhunen noted that such a spacecraft could be used in multiple different ways [86]: for example, missions travelling out of the Solar system, where it should be possible to achieve final speeds of greater than around $50 \mathrm{~km} / \mathrm{s}$, or alternatively spiraling in to the centre of the Solar system towards, for example, Mercury, by pitching the sail correctly. Mengali and Quarta then used the idea of the electric sail to study possible non-Keplerian orbits aimed at the observation of the Sun's polar regions [87], providing an overview of the electric sails capabilities in terms of the required values of orbital plane position, view angle and thrust angle for a given orbital period and sail performance characteristic acceleration. Ref. [87] also outlined optimal orbit transfer trajectories, by minimizing the flight time necessary to transfer the spacecraft from a given parking orbit to the desired final non-Keplerian orbit, and further, compared the relative performances of the electric sail and the traditional solar sail in terms of their dimensionless propulsive acceleration components in maintaining a displaced non-Keplerian orbit of given characteristics. This analysis suggests that the electric sail is an interesting alternative to a solar sail, although it is hard to discern whether it could yet be deemed superior, given the quite different nature of the two systems.

Another potential alternative is that of the compound solar sail, which uses two reflectors. There are two different types of compound sail generally suggested - namely, the Simple Solar Photon Thruster (SSPT) and the Dual Reflection Solar Photon Thruster (DRSPT). Here just the former is discussed: the solar photon thruster as proposed by Robert Forward [88], which is fundamentally simpler (for reference, the latter is also examined in Ref. [88]). The 
idea of the SPT is to have two sails: one of which is a large collecting sail, always normal to the Sun, which directs the photons onto a smaller, steerable collector thruster. Thus the function of collecting the solar photons was separated from that of reflecting them, allowing the sail to collect the maximum amount of light possible at all times and yet allow it to be redirected in the desired direction, thus overcoming the obvious failing of the standard solar sail. Such a concept has been considered by several authors, for example Mengali \& Quarta [89] and Dachwald \& Wurm [90]. Like electric sails, employing the concept in the context of highly non-Keplerian orbits is still in its infancy, although as an extension of the analysis already presented by Forward [88], Matloff has considered the SPT as a terrestrial pole sitter, showing that an SPT (with what was claimed to be an achievable areal mass thickness of 2 $\mathrm{g} / \mathrm{m}^{2}$ and thruster and collector reflectivities of 0.9 ) could be stationed above latitude $45^{\circ}$ at a distance of around 60 Earth radii [91].

A third novel system proposed by Peck [92], that of Lorentz-augmented orbits, is like the solar sail in the sense that it is propellantless. A Lorentz-augmented orbit (LAO) exploits the interaction between the Earth's geomagnetic field and an electrostatic charge that can be built up on a satellite by using electrical power. This interaction produces an electrodynamical propulsion - via the terms of the Lorentz force experienced by a particle of charge $q$ (in Coulombs) moving through a magnetic field $\boldsymbol{B}$, i.e. $\boldsymbol{F}_{L}=q \boldsymbol{v}_{r} \times \boldsymbol{B}$ where $\boldsymbol{v}_{r}$ is the particle velocity with respect to the magnetic field. Streetman \& Peck took the concept of LAO's and showed how, by consideration of the equations of motion governing the motion of satellites experiencing the Lorentz force, new types of Earth-synchronous orbits arise [93]. Further, it was shown that for polar-orbiting satellites, a constant electrical charge can create arbitrary changes in the right-ascension angle - which can be used to produce single-orbit repeat groundtrack low Earth orbit satellites - and that in the equatorial case, the longitude of perigee can be manipulated via a constant electrostatic charge, allowing for the creation of an Earth-synchronous type of orbit.

There are some other different and exotic types of low thrust propulsion. Two more examples are that of nuclear electric propulsion, and the magnetoplasma sail. The former is simply a direct extension of the concept of solar electric propulsion but with a different power source, so the work as discussed on SEP above can be thought of as relevant for nuclear electric propulsion.

The latter, however, has been considered in some detail - see, for example, the MagSail concept outlined by Andrews, Zubrin and co-authors (Refs. [94, 95, 96, 97]), and the mini-magnetospheric plasma propulsion device (M2P2) proposed Winglee et al. [98]. More recently, Kajimura et al. have carried out numerical simulations of a magnetoplasma sail by using a three-dimensional hybrid code [99]. However, magnetoplasma sails have not been considered a great deal in the context of highly non-Keplerian orbits, and thus are not considered further here. But, as this subsection (and Section 3.4) has shown, consideration of low-thrust propulsion technologies beyond that of just conventional solar sails and SEP is increasing and thus in future more work on highly non-Keplerian orbits using these technologies will inevitably follow. 


\subsection{Extensions of the model}

As one might expect, the research outlined above has been or is being extended in numerous different ways to examine new aspects of the problem or existing aspects in a novel way. In this subsection, a few such branches are outlined briefly.

Much of the work on artificial equilibrium points with solar sails primarily considers the Sun-Earth case, although Ref. [100] investigated a new family of displaced solar sail orbits near the Earth-Moon libration points, which extended the work of Vonbun [101] and Farquhar [14] on the dynamics of artificial bodies in proximity to the classical Lagrange points. More recently Simo \& McInnes [102,103] have also considered solar sail periodic orbits in the Earth-Moon restricted three body problem. Here the dynamics are quite different from the Earth-Sun system in that the sun line direction constantly changes in the rotating frame, rotating once per synodic lunar month. Using an approximate, first-order analytical solution to the non-linear non-autonomous ordinary differential equations, periodic orbits can be constructed that are displaced above the plane of the restricted three-body system. This new family of orbits have the property of ensuring visibility of both the lunar far-side and the equatorial regions of the Earth, and can enable new ways of performing lunar telecommunications. Ref. [103] found that for a given displacement distance above/below the Earth-Moon plane it is easier by a factor of order 3.19 to do so at $\mathrm{L}_{4} / \mathrm{L}_{5}$ compared to $\mathrm{L}_{1} / \mathrm{L}_{2}$ - i.e. for a fixed sail acceleration the displacement distance at $\mathrm{L}_{4} / \mathrm{L}_{5}$ is greater than that at $\mathrm{L}_{1} / \mathrm{L}_{2}$. Additionally, displaced $\mathrm{L}_{4} / \mathrm{L}_{5}$ orbits are passively stable, making them more forgiving to sail pointing errors than highly unstable orbits at $\mathrm{L}_{1} / \mathrm{L}_{2}$. The drawback of the new family of orbits was shown to be the increased telecommunications path-length, particularly the Moon- $\mathrm{L}_{4}$ distance compared to the Moon- $\mathrm{L}_{2}$ distance. The same authors have also compared the dynamics of displaced orbits in the two and three-body Earth-moon problems via an asymptotic analysis for large and small accelerations [104], as well as analyzing the case of displaced lunar orbits using hybrid propulsion [62]. Additionally, much research on libration point orbits in the vicinity of the Moon has been considered by researchers at Purdue University - this is discussed in Section 4.1 in the context of mission applications, but see, for example, Wawrzyniak \& Howell [105], and Ozimek and co-authors [106], amongst others.

The work discussed in Section 3 up to this point only considers the CRTBP, but there is also a growing body of literature on generalisation to the more realistic elliptical restricted three-body problem (ERTBP). Baoyin \& McInnes [107] showed that the three-dimensional equilibrium surfaces from the CRTBP do not exist in the ERTBP, because of the stretching of the coordinate frame in the system plane (the out-of-plane coordinate remains unstretched, however). Instead planar two-dimension equilibrium curves exist in the ERTBP, embedded in the threedimensional, non-uniformly rotating, pulsating coordinate system. Biggs et al. [108] illustrated that a solar sail does not track a periodic reference orbit obtained from the CRTBP adequately, and detailed methods for constructing better reference trajectories based on numerical continuation, by using the eccentricity $e$ as a perturbation parameter to find a natural orbit in the ERTBP - with such orbits significantly improving the tracking error of the solar sail. 
Of course, having considered the two-body and three-body cases, there is scope for expanding the study of highly non-Keplerian orbits within a four-body formulation. However, although there is some work in the literature on classical Keplerian orbits in such a set-up (e.g. Howell and Spencer's study of periodic orbits in the restricted fourbody problem [109], this is still a relatively un-explored field from a highly non-Keplerian orbit context, although Ceccaroni et al. are considering such problem for the identification of asteroid-centered NKO's [110].

\section{Mission Applications}

Potential missions using highly non-Keplerian orbits can be broken down into categories depending on the mission application. As discussed throughout, work on such orbits has mostly focused primarily on Earth-centered trajectories, although some authors have considered individual applications of highly non-Keplerian orbits outwith the Earth's influence. In this section some examples of potential applications are considered.

\subsection{Telecommunications}

Various authors have considered the use of highly non-Keplerian orbits to enable novel telecommunications concepts, either in the context of terrestrial communications or for human exploration of the Solar System. On the former, there is a substantial body of literature - for example, Dittberner et al. [111], and Diedrich \& Mulligan [112], who discussed using solar sails in artificial Lagrange orbits for polar communications with high latitude users (e.g. science stations and shipping), as well as for communication with other space assets, like a constellation of polar orbiting satellites. More recently, Baig \& McInnes [113] carried out an analysis into the possibility of generating displaced non-Keplerian periodic orbits around geostationary points in the solar sail two-body problem, showing that such a family exist at linear order. In turn this work proved that the original proposal of Forward [12, 13] (to use such orbits to deploy satellites into orbits displaced above or below the usual geostationary ring, whilst retaining the same orbital period) was correct, albeit that the actual displacement distances possible are modest.

In terms of telecommunications for exploration, McInnes \& Simmons discussed the use of displaced Sun-centered non-Keplerian orbits to avoid communications blackouts in interplanetary communications [17]. More recently McKay and co-workers [43] outlined a concept for an Earth-Mars communications relay that used such orbits to hover above the orbital plane of Mars in order to relay signals between the two bodies during periods of occultation by the Sun, illustrated in Figure 21, and thus ensure continuous communications between Earth and assets on the surface of Mars. 


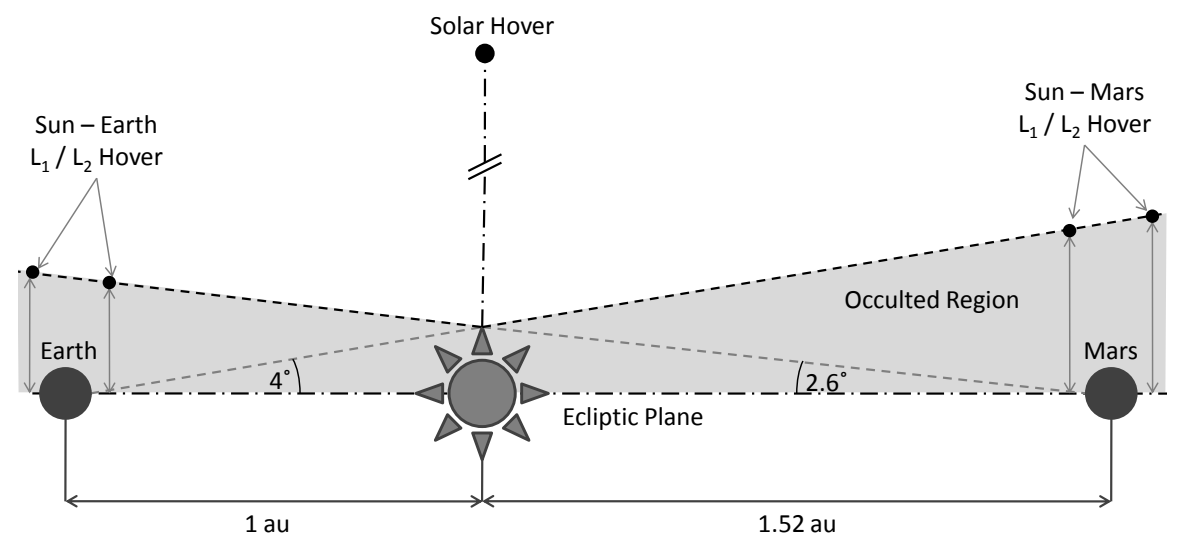

Fig. 21: Mars-Earth communications relay architecture options out of the Ecliptic plane. From Ref. [43].

The technology requirements of such an array are largely determined by the field-of-view exclusion zone about the Sun, which is dependent on how close to the limb of the Sun radio signals can be transmitted without interference from the solar plasma. For design optimisation purposes a spacecraft in proximity of Mars is preferred as the long slant range back to Earth can be compensated for through the use of a large Earth-based antenna. By considering the highly non-Keplerian orbit regions about Mars accessible to a SEP spacecraft of $m=1000 \mathrm{~kg}$ and maximum thrust $300 \mathrm{mN}$, the authors illustrated how such a relay spacecraft could be stationed up to approximately $0.176 \mathrm{AU}$ above or below the poles of Mars, and thus enable such a relay assuming the 4 degree field-of-view exclusion angle that Xband communication would require. Considering K-band communications - which would reduce the required fieldof-view exclusion angle from 4 degrees to just 1.5 degrees - would require a thrust of about $200 \mathrm{mN}$ for the same relay architecture. Other options were also considered, such as using a second spacecraft, with the advantages of such a setup being that the thrust requirements are significantly reduced by reducing the need to displace the spacecraft so far out of the orbital plane, whilst maintaining good polar coverage (or, if desired, allowing access to observation of/communication with equatorial regions instead). Recently, McKay and co-authors analyzed the potential of hybrid propulsion within the Mars-Earth communications relay concept, showing that the addition of a small solar sail to an SEP thruster can reduce the amount of thrust required from the SEP component, but is only practical for the specific case where the relay spacecraft is stationed in a polesitter orbit during the Martian summertime and communicates with Earth via Ka-band [114].

Other authors have considered highly non-Keplerian orbits for telecommunications purposes, particularly in the vicinity of the Moon, as discussed previously [100, 102, 103]. Wawrzyniak \& Howell [105] considered the use of such orbits for a lunar telecommunications relay, calculating the instantaneous equilibrium surfaces in the EarthMoon system using current and future sail technology. These surfaces can be exploited as starting points for designing orbits that always remain in view of the Lunar South Pole (LSP), in order to enable continuous communications with Earth. Similarly, by using Hermite-Simpson and seventh-degree Gauss-Lobatto collocation schemes Ozimek and co-authors [106] considered options for constant lunar south pole coverage using displaced 
periodic orbits in the Earth-Moon restricted three-body problem for a solar sail. In the process it was shown that orbits near the Earth-Moon $\mathrm{L}_{2}$ point yield the best coverage results. The same authors have also studied in-depth the design and modelling of optimal low-thrust lunar pole-sitter missions and trajectories using a thruster similar to Deep Space 1's NSTAR [115, 116]. Grebow et al. [117] identified feasible orbits for LSP coverage from the halo, vertical and butterfly families in the vicinity of the Earth-Moon $L_{1}$ and $L_{2}$ points in the CRTBP, studying nine different orbits (with periods ranging from 7 to 16 days) occupied by two phase spacecraft either in a single orbit or in a combination of two different orbits.

\subsection{Earth Observation}

As was referred to previously, many authors have considered the use of highly non-Keplerian orbits to enable "polesitter"-type orbits $[9,35,91]$. Such orbits are desirable because of their ability to provide constant observation of the polar regions of the Earth, which may be useful for, for example, providing real-time hemispherical, low resolution imaging of the poles for climate science and synoptic forecasting. An alternative use would be for line-ofsight communications to polar assets such as Antarctic science stations and (new) polar shipping lanes. Such a mission can be considered from either the point of view of occupying an artificial equilibrium point, or performing a periodic orbit about an AEP.

In the former case, it has been found that it is not actually possible to have a solar sail permanently fixed above a pole of the Earth [36], as had been originally envisaged. In addition, the volume of space within which equilibria are possible on the night-side of the Earth is severely constrained with a realistic solar sail, as shown previously (see Figure 14). Here, an advantage of SEP over solar sailing becomes clear, in that there are no forbidden regions (due to the orientation constrained nature of the solar sail requiring that it thrust away from the Sun) - meaning that there are areas around both $\mathrm{L}_{1}$ and $\mathrm{L}_{2}$ which are accessible to an electric propulsion system that are not accessible to a sail (not forgetting, of course, that SEP is constrained by reaction mass, unlike solar sailing). McKay et al. [43] showed that a 1000kg SEP spacecraft could hover 0.0085AU (approximately 1.28 million $\mathrm{km}$ ) directly above the Earth's poles with a maximum thrust of $300 \mathrm{mN}$.

Alternatively, a potential solar sail PoleSitter mission could consist of an orbit considered such that the solar sail is displaced high enough above the ecliptic plane on the day-side of the Earth to be stationed directly over the north pole at the summer solstice [118]. This can be done with a sail of non-ideal optical reflectivity of 0.85 and $\beta=$ 0.099 (equivalent to a characteristic acceleration of $0.55 \mathrm{mms}^{-2}$ ), which could be stationed at an equilibrium point 612 Earth radii directly above the North Pole at the summer solstice. From this vantage point a constant daylight view of the Arctic is available at the summer solstice. Such a vantage point could also prove useful in the context of line-of-sight telecommunications to high-latitude users. However, the equilibrium location is fixed relative to the Sun and the Earth, and therefore six months later at the winter solstice the solar sail is no longer directly over the North Pole, due to the fixed tilt of the Earth's polar axis as the Earth orbits the Sun. From this new vantage point a constant view of high latitude regions would still be available, though. 
In the latter case, in Ref. [70] the case of periodic orbits about equilibrium points in the Sun-Earth rotating frame high above the ecliptic plane was considered (as described in Section 3.5), with families of periodic orbits being identified. Such orbits are also appropriately positioned for the constant viewing of Earth's polar regions: with orbit periods of one year, and by correctly timing the orbits in order to narrow the annual variation in the elevation angle of the sail above the local horizon, the seasonal effect of the variation of Earth's axis of rotation can be countered for, as displayed in Figure 22.

Ref. [70] showed that this can be done by enabling periodic orbits around a reference equilibrium point requiring a sail $\beta=0.05174$ (equivalent characteristic acceleration $0.3 \mathrm{mms}^{-2}$ ). However, these orbits are inherently unstable and thus a controller is required in order to maintain the spacecraft on them for long periods of time, although it was suggested that by numerically continuing the families of orbits beyond the region where the linear terms dominate then regions of stable periodic orbits may be found.

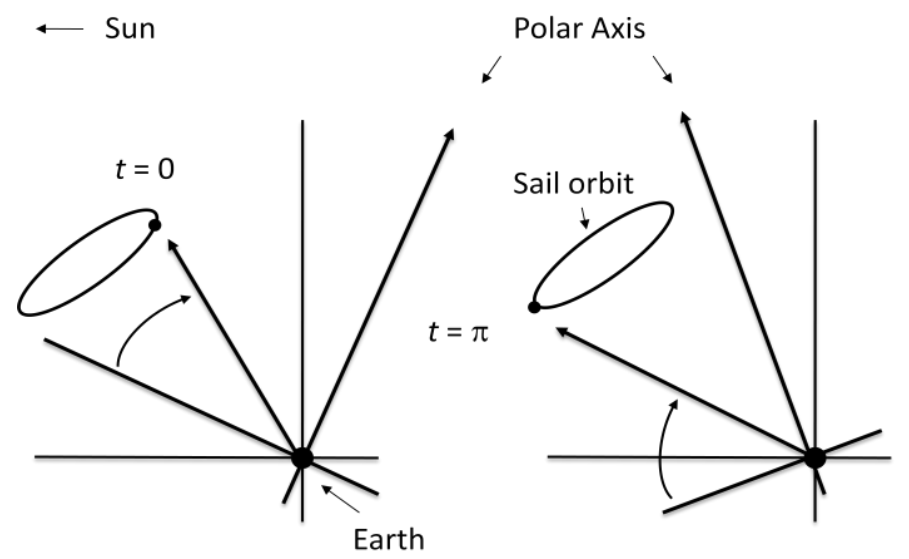

Fig. 22: One-year orbits that counter the seasonal variation in the Earth's axis of rotation, with the sail position shown schematically (in the rotating frame) in wintertime (left) and summertime (right). From Ref. [70].

\subsection{Planetary Science}

Spilker [119] expanded upon the research of Refs. [11] and [41] regarding in-situ observation of Saturn's rings. The study and understanding of the dynamics of the rings is of high interest in planetary science due to their complex structure and poorly understood formation and evolution mechanisms. Particularly of interest would be studies of the microphysics of individual particles on the centimeter level, and other small scale phenomena (in the region of meters to a few kilometers), such as particle agglomeration behavior. A potential candidate displaced nonKeplerian orbit above Saturn's ring plane could provide the opportunity for a spacecraft to "hover" over a nominal ring particle or cluster of particles, and track their behavior over an extended period. Spilker detailed the initiation and use of a non-Keplerian orbit for studying the rings, estimating that, for a $100 \mathrm{~kg}$ spacecraft offset $3 \mathrm{~km}$ from the rings at $r=125,000 \mathrm{~km}$, the required force is about $6 \mathrm{mN}$. The wide range of different 2-body orbit families outlined 
previously would allow flexibility with the choice of spacecraft orbit - for example, thrust contours of minimum acceleration can be plotted for a given hover altitude, or thrust contours equating to a specific orbital period that would allow the craft to remain above the same point in the ring can be created.

In other work Sawai, Scheeres \& Broschart analyzed the control of a spacecraft hovering over a rotating body such as a comet or asteroid [120]. Broschart \& Scheeres [121] extended this work in the first instance by considering the case of using continuous control thrust to hover above an asteroid, and investigated the stability of realistic hovering control laws in both the body-fixed and inertial reference frames. The authors also presented a case study of hovering above Asteroid (25143) Itokawa, which was the target of the Hayabusa mission. More recently the same authors have derived sufficient conditions for local and global boundedness of spacecraft motion inside a prescribed region subject to a dead-band hovering thrust control law in time-invariant Lagrangian dynamics systems [122]. In a similar study Morrow et al. [123] considered the use of solar sails operating in close proximity about asteroids, showing that both hovering and stable sun-synchronous orbital options are possible. Such techniques that can enable a spacecraft to remain stationary above a given point may be extremely useful in observational studies, and could be adapted for other small celestial bodies - for example, comets, or dwarf planets such as Ceres.

\subsection{Observational Astronomy/Astrophysics}

There are various different examples of potential uses for highly non-Keplerian orbits in observational astronomy and astrophysics.

One well studied idea is that of the GeoStorm mission concept, as referred to in Section 1.2. GeoStorm originated in the late 1990's after the National Oceanic and Atmospheric Administration (NOAA) asked the Jet Propulsion Laboratory (JPL) if it was possible to improve the warning time of impending space weather events, such as geomagnetic storms, via the application of emerging new technologies such as solar sails and micro-spacecraft.

Magnetic storms - bombardments of high-energy particles caused by solar coronal mass ejections (CME's) and solar flares - pose a high risk to electrical and telecommunications equipment at both the Earth's surface and in the lower atmosphere. Currently, probes orbiting the Earth-Sun $\mathrm{L}_{1}$ point can provide approximately 30 minutes advance warning of an approaching CME.

The aim of the resulting 1999 ST-5 GeoStorm mission proposal was to use a solar sail of characteristic acceleration $0.169 \mathrm{mms}^{-2}$ to access an artificial displaced orbit at a point sunward of $\mathrm{L}_{1}$ (0.993AU from the Sun), instead maintaining station at $0.984 \mathrm{AU}$ [38]. This would increase the warning time of an approaching magnetic storm by a factor of approximately 3. A nominal trajectory for GeoStorm involved a transfer time of 3 months from LEO to $\mathrm{L}_{1}$ on a ballistic trajectory and then a sail trajectory of 192 days to move from $\mathrm{L}_{1}$ to sub- $\mathrm{L}_{1}$ [39]. The ST-5 design was not chosen by NASA for flight demonstration; however, it did highlight the performance potential. Further work by JPL [38] involved an improved solar sail design that would allow a craft of mass approximately 95kg and 
characteristic acceleration $0.438 \mathrm{mms}^{-2}$, to maintain station at $0.974 \mathrm{AU}$, increasing the warning time yet further (by another factor of 2 compared to the 1999 mission proposal).

McKay et al. [43] applied the same principle to a continuous low-thrust SEP spacecraft of $m=1000 \mathrm{~kg}$, showing that although an orbit at $0.974 \mathrm{AU}$ would not be achievable due to the high mass of the craft, $300 \mathrm{mN}$ of thrust would make it possible to station at approximately $0.981 \mathrm{AU}$. This would allow for a geomagnetic storm warning time of upwards of 90 minutes. Of course, given the finite amount of propellant stored on board the SEP spacecraft, this position could only be maintained for approximately 3 years, necessitating future missions to re-establish a warning post. In theory a solar sail could remain on-station for an infinite amount of time, although in practice degradation of the reflective surface and on-board electronics would eventually terminate the mission.

Sauer suggested that a more extensive application of the GeoStorm warning concept would involve a group of four solar sails, placed in a diamond arrangement adjacent to $\mathrm{L}_{1}$ on a halo orbit [124]. Such a configuration would allow for multi-point measurements of the solar environment, potentially allowing several science objectives to be completed, such as measuring the spatial variation in convected and propagating waves and shocks in the solar wind and discovering the properties of solar wind turbulence as a function of separation and time. Further, Sauer detailed a conceptual diagram of a possible $\mathrm{L}_{1}$ Diamond concept [124], with the origin of the diamond being offset from $\mathrm{L}_{1}$ towards the Sun by a distance $\mathbf{d}$. The diamond consists of a monitoring position at the chosen separation distance directly above the diamond origin, a monitoring position located at the separation distance radially inwards from the diamond origin, and 2 side points in the ecliptic separated from the diamond origin by half the separation distance. As Sauer notes, there are several ways to configure the diamond formation, with the most obvious choices being related to the characteristic acceleration required by each spacecraft. If all the sails are to have the same characteristic acceleration then there may be large differences in the separation distances of the various spacecraft, meaning that it may be simpler to keep all the separation distances the same and require the spacecraft to each maintain their own specific acceleration.

Formation flying is advantageous because it allows the opportunity to use small groups of less expensive or complex satellites as opposed to a single large satellite, and thus mitigates the risk of a catastrophic failure which would prematurely end the mission. Outwith the GeoStorm concept, other authors have considered the general problem of solar sail formation flying around displaced non-Keplerian orbits. As discussed in Section 2.1.5, Gong, Baoyin and Li [48] considered such a case in the context of orbit control, and in other work Biggs \& McInnes [125] proposed, in the ERTBP, a solar sail formation flying high above the $\mathrm{L}_{1}$ libration point for remote sensing of the polar regions of the Earth.

A quite different example of work on NKO's in the context of observational astrophysics is provided by Kezerashvili and Vázquez-Poritz, who considered the effects of General Relativity on the bound motion of solar sails for both orbits within the plane of the Sun as well as non-Keplerian orbits $[126,127]$. The simultaneous effect 
of the curvature of spacetime and the solar radiation pressure are sufficient to lead to deviations from Kepler's third law. Further, the work in Refs. [126, 127] also studied and quantified deviations from Keplerian motion due to frame dragging, the gravitational multipole moments of the Sun, a possible small net electric charge on the Sun, and a small positive cosmological constant, and suggested that a near-solar mission could provide confirmation of such phenomena. It is worth pointing out, however, that such effects may be masked somewhat by effects relating to the solar sail itself - for example, poorly quantified optical surface degradation or guidance and control errors.

\subsection{Geoengineering}

A relatively new avenue of research is to consider the use of highly non-Keplerian orbits for geoengineering purposes. Geoengineering has come to refer to the concept of deliberately manipulating the Earth's climate in order to counteract or mitigate the effects of global warming from greenhouse gas emissions. Geoengineering is a deep and complex field within itself, with, generally speaking, two main types of suggestion: those that try to deal with the increasing levels of $\mathrm{CO}_{2}$ in the Earth's atmosphere (i.e. carbon sequestration techniques), and those that attempt to cool the Earth by management of solar radiation levels. Ref. [128] neatly summarizes the prospects and problems of engineering the climate by such methods.

One space-based, large-scale proposed solution is that of a solar shield, or mirror, which could be used as an occulting disc to reduce the amount of sunlight striking the Earth. Such an object would be placed at the Sun-Earth $\mathrm{L}_{1}$ Lagrange point. It appears that James Early was one of the first to suggest such a proposal [129], which involved a $2000 \mathrm{~km}, 10$ micron think glass shield made of Moon rock which would reduce solar insolation by about $2 \%$. At about the same time Seifritz also suggested a similar scheme [130], although his shield would be made of aluminum and would compensate for a temperature increase of $2.5 \mathrm{~K}$ on Earth with a disc approximately $2400 \mathrm{~km}$ in diameter. Other authors have since published work on a similar vein [131, 132, 133].

Further, the concept of highly non-Keplerian orbits and displaced artificial libration points has allowed authors to expand upon the original solar shield concept. In considering the three-body mechanics of the problem, Ref. [134] optimized the shield location (placing it sunward of the Sun-Earth $\mathrm{L}_{1}$ point) and thus was able to minimize the mass of shield required, although at $10^{11} \mathrm{~kg}$ required to offset a global temperature enhancement of $2 \mathrm{~K}$, the engineering challenge is still immense. In order to mitigate the chances of the loss of a single large disc, McInnes suggested using large numbers of smaller discs [135].

Angel [136] outlined a concept of implementing a sunshade as a cloud of many small (meter-sized) autonomous spacecraft, in order to block $1.8 \%$ of the solar radiation flux. The spacecraft cloud would be located at a point near the Sun-Earth $\mathrm{L}_{1}$ point in an orbit with the same 1-year period as the Earth, in-line with the Sun at a distance of greater than about 1.5 million $\mathrm{km}$. From this distance, the penumbra shadow covers and thus cools the entire planet. Simply creating the sunshade from a large number of dust particles ignores the fact that such an orbit would be unstable and the cloud would dissipate quite quickly without any stabilizing control force applied. However 
individual spacecraft could maintain the orbits with active station-keeping, with stabilizing forces obtained by modulating solar radiation pressure. The total mass of the cloud would be approximately 20 million tonnes and cost a few trillion dollars to develop and deploy.

More recently Biggs and McInnes [137] showed that a solar sail may stabilize its motion (in the Lyapunov sense) about an artificial libration point using only small variations in its lightness number $\beta$, despite the fact it is only $\beta$ controllable in the plane, rather than relying on pitch and yaw angle active control methods. Such work is important in the context of solar shields for space-based geoengineering, given that active control of the attitude of such a large structure (for station-keeping purposes about such unstable artificial libration points) is a very challenging engineering problem and hence passive stabilization can reduce the complexity of such schemes.

\section{Summary}

As has been demonstrated, using continuous low thrust propulsion to enable highly non-Keplerian orbits (termed such when the magnitude of the acceleration applied is of approximately equal magnitude to that of the gravitational acceleration experienced) is a rich vein of astrodynamics research, both currently and in broader historical terms. It has been shown here that the problem has been considered from a number of different viewpoints, in terms of propulsion systems (e.g. solar electric propulsion (SEP), solar sails, hybrid SEP/sail and other more exotic methods) and considerations of the $\mathrm{N}$-body problem (e.g. two-body, three-body circular restricted and elliptical restricted, four-body restricted). Many authors have analyzed the dynamics, stability and control of such orbits, and determined orbits that connect them with other orbits, both Keplerian and non-Keplerian. The problem has been considered for various locations across the Solar System, for example in the Sun-Earth and Earth-Moon problems, and at Mars and Saturn, amongst others. It has also been shown that such orbits could have a vast range of potential applications, including but not limited to telecommunications, Earth observation, planetary science, observational astrophysics and space-based geoengineering.

It is clear, however, that work still has to be done to transform the steadily-growing body of literature on highly nonKeplerian orbits from interesting theory into actual, practical missions.

Firstly, as discussed in Section 3, a lot of this literature concerns itself with the rather idealized circular restricted three-body problem. Analysis of highly non-Keplerian orbits in the more realistic elliptical restricted three-body problem is still in its relative infancy, although as shown some authors have already begun considering the problem. Clearly increasing the knowledge base in this area, and incorporating other non-ideal effects into the model will be important in proving, refining and maturing the concept for the "real world".

Secondly, improving the technology will increase the feasibility of using highly non-Keplerian orbits in missions. The more thrust that an ion engine can generate, the greater the gravity gradient that can be compensated for and hence the more opportunities there are for applying non-Keplerian orbits. Additionally, in some aspects, engineering 
capability does not necessarily match theoretical requirements. For example, as discussed in Section 2, the variable mass nature of solar electric propulsion means that the acceleration of a body will increase as propellant is burned off. Thus, ideally, the thrust level of the engine would be smoothly throttled down in small increments in order to maintain the same relative position over the duration of a non-Keplerian orbit mission - if the thrust level remains the same but the mass decreases, then the acceleration will subsequently increase and the spacecraft will perform a rising helical orbit (as opposed to the intended closed circular orbit). However, such fine throttle control is beyond current capabilities, although very coarse throttle control is not, and finer control may be possible in the near future. An alternative possible solution may be to operate the thruster in a pulsed mode, such that there are short periods when the thruster is not operating, and thus providing a way to modulate the average thrust over the duration of the orbit by varying the duration of the pulses.

In terms of solar sails and their engineering requirements the recent successful deployment of JAXA's IKAROS sail (and subsequent observations of its acceleration by photon pressure) will surely spur the development of future solar sails, allowing for the design, creation and deployment to be refined over successive missions. The lessons learned with each attempt will potentially increase the performance of solar sails over time, making some of the missions suggested previously more feasible. Further, the concept of the hybrid sail may prove both a useful stepping stone in solar sail development, by allowing the performance of smaller prototype sails to be enhanced with proven, wellunderstood SEP technology, as well as possibly being the natural successor to both SEP and solar sail missions in combining the benefits of both propulsion technologies. And of course, as briefly touched on, there are other means of producing continuous thrust that could also be used to generate highly non-Keplerian orbits - with this area still being a relatively untapped avenue of research.

Finally, there must be a desire to want to perform highly non-Keplerian missions in the first place. It must be made obvious to national space agencies and industrial/commercial space companies why they are of interest, what novel things they can do (particularly if they cannot be achieved by other means) and, especially in the industrial/commercial sector, what competitive advantages they could provide. This review has already highlighted some of the proposed applications for highly non-Keplerian orbit missions - applications which span a broad range of categories - but there are surely many more still to come to light. One can envisage the whole field as a feedback loop, with novel ideas proving the driver for new research which will ultimately increase the opportunities for more missions and eventually more ideas. The signs are that national space and government agencies, as well as private companies, have already shown interest in some of the concepts and missions proposed: engaging with these bodies will be vital in transferring the concept from theoretical research to practical applications over the coming years, but it seems clear that the concept is very promising and yet to be fully exploited.

\section{Acknowledgments}

C. McInnes would like to acknowledge European Research Council Project 227571 (VISIONSPACE). 


\section{Author Biographies}

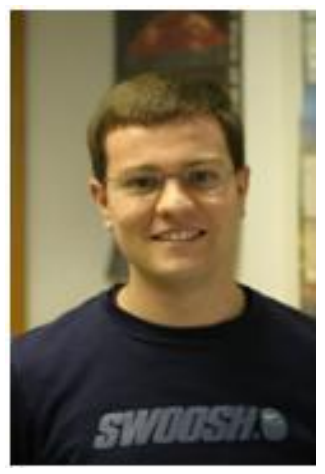

Dr. Robert McKay joined the Advanced Space Concepts Laboratory at the University of Strathclyde as a Research Assistant, having obtained his PhD. originally in solar astrophysics and fusion plasma physics. He assumed a study technical lead role on a collaborative project for an ESA General Studies Programme study, titled Gravity Gradient Compensation Using Low Thrust, High Isp Motors. This study involved modeling highly nonKeplerian orbits throughout the Solar System for both solar sail and solar electric propulsion spacecraft, and determining novel missions that are enabled by these orbits. McKay's research on non-Keplerian orbits can be viewed in 2 conference papers, with another 2 peer-reviewed journal papers currently in press. His research is currently being funded primarily by industry.

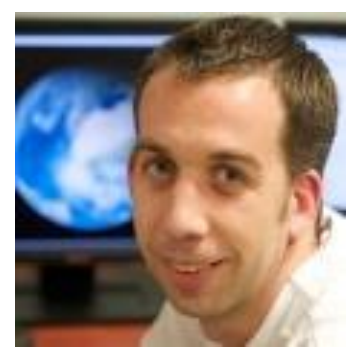

Dr. Eur. Ing. Malcolm Macdonald is an Associate Director of the Advanced Space Concepts Laboratory at the University of Strathclyde, which he joined in 2008 following three years working in the European space industry on a range of missions throughout the project life cycle. He has a diverse research track-record, seeking to develop and apply advanced space mission systems, challenging conventional ideas, and enabling significant change in the near term. His research ranges from advanced astrodynamics to the study of 
technology requirements for a range of future missions, and the development of nanospacecraft. Macdonald's research is reported in over 25 peer reviewed journal papers, including prize-winning publications, such as the Royal Aeronautical Society Ackroyd Stuart Propulsion Prize (2003). Since joining the University of Strathclyde, his research has funded by the European Space Agency, a range of industrial partners (EADS Astrium, Clyde Space Ltd.) and research councils (EPSRC, STFC, CEOI/NERC).

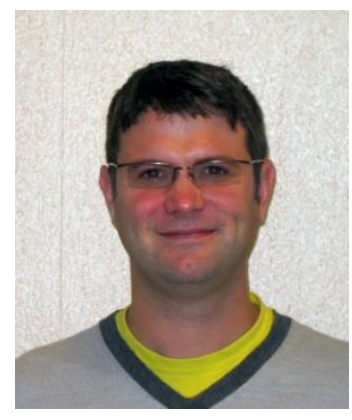

Dr. James Biggs is an Associate Director of the Advanced Space Concepts Laboratory. His research interests encompass the application of mathematics which include geometric control, nonlinear dynamics, chaos and control of chaos, non-holonomic mechanics, complexity science and its applications to motion planning and the control of underwater vehicles, unmanned air vehicles, and spacecraft. He has worked as technical lead for ESA's Advanced Concepts team on relative satellite motion which designing controls for low Earth orbit formation flying. Biggs also undertook post-doctoral research in the area of solar sail non-Keplerian orbits in the restricted three-body problem while at the University of Strathclyde. He is a reviewer for several international journals, including IEEE Transations on Automatic Control, Journal of Mathematical Control, Systems and Signals, Mathematical Reviews, and the Journal of Guidance, Control and Dynamics. Biggs' research is reported in 9 peer-reviewed journal papers, and 13 conference papers. 


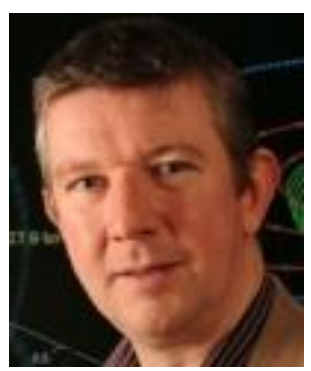

Prof. Colin McInnes is Director of the Advanced Space Concepts Laboratory at the University of Strathclyde. His work spans highly non-Keplerian orbits, orbital dynamics and mission applications of solar sail spacecraft, control of multiple spacecraft using artificial potential field methods, future concepts such as space-based geo-engineering and is reported in 120 peer-reviewed journal papers. Recent work is exploring new approaches to spacecraft orbital dynamics at extremes of spacecraft length-scale to underpin new spacederived products and services. McInnes has been the recipient of national and international awards including an inaugural Philip Leverhulme Prize from the Leverhulme Trust (2001), the Royal Aeronautical Society Pardoe Space Award (2000) and the Ackroyd Stuart Propulsion Prize (2003). More recently he was awarded a Leonov medal by the International Association of Space Explorers in September 2007. Over the last ten years McInnes' work has been funded by a diverse range of international partners including Research Councils (ERC, EPSRC, STFC), the European Space Agency and industry (EADS Astrium, Lockheed-Martin ATC).

\section{References}

[1] Macdonald M., McInnes, C. R. "GeoSail; An Enhanced Magnetosphere Mission, Using a Small Low Cost Solar Sail”, Proceedings of $51^{\text {st }}$ International Astronautical Congress, IAC Paper 00.W.1.06, Rio de Janeiro, Brazil, Oct $2^{\text {nd }}-6^{\text {th }}$, 2000

[2] McInnes, C. R., Macdonald, M., Angelopolous, V., and Alexander, D., "GEOSAIL: Exploring the Geomagnetic Tail Using a Small Solar Sail”, Journal of Spacecraft and Rockets, Vol. 38, No. 4, pp. 622-629, 2001

[3] Macdonald, M., Hughes, G.W., McInnes, C. R., Lyngvi, A., Falkner, P., Atzei, A., “GeoSail: An Elegant Solar Sail Demonstration Mission”, Journal of Spacecraft and Rockets, Vol. 44, No 4, pp. 784 - 796, 2007

[4] Oyama, T., Yamakawa, H., and Omura, Y., “Orbital Dynamics of Solar Sails for Geomagnetic Tail Exploration”, Journal of Spacecraft and Rockets, Vol. 45, No. 2, pp. 316, 2008

[5] Macdonald, M., McKay, R. J., Vasile, M., and Bosquillon de Frescheville, F.., "Extension of the Sun-Synchronous Orbit", Journal of Guidance, Control, and Dynamics, in press, 2010

[6] Oberth, H., Ways to Spaceflight, Verlag, Berlin, 1929 (also NASA TTF-622)

[7] Dusek, H. M., "Motion in the Vicinity of Libration Points of a Generalized Restricted Three-Body Model", Methods in Astrodynamics and Celestial Mechanics: Progress in Astronautics and Aeronautics, Vol. 17, pp. 37-54, 1966 
[8] Austin, R.E., Dod, R.E. and Terwilliger, C.H., "The Ubiquitous Solar Electric Propulsion Stage", Acta Astronautica, Vol. 4, No. 5-6, pp. 671-694, 1977

[9] Driver, J. M., “Analysis of an Arctic Polesitter”, Journal of Spacecraft and Rockets, Vol. 17, No. 3, pp. 263-269, 1980

[10] Lasswitz, K., Two Planets, Southern Illinois University Press, ISBN 0-8093-0508-9, 1971

[11] Nock, K. T., "Rendezvous with Saturn's Rings", Anneaux des Planetes, IAU Colloquium No. 75, Cepadues Editions, Toulouse, pp. 743-759, 1984

[12] Forward, R. L., "Light Levitated Geostationary Cylindrical Orbits”, Journal of the Astronautical Sciences, Vol. 29, No. 1, pp. $73-80,1981$

[13] Forward, R. L., "Light Levitated Geostationary Cylindrical Orbits Using Perforated Light Sails", Journal of the Astronautical Sciences, Vol. 32, No. 2, pp. 221-226, 1984

[14] Farquhar, R., "Limit Cycle Analysis of a Controlled Libration-Point Satellite", The Journal of the Astronautical Sciences XVII, Vol. 17, No. 5, pp. 267-291, 1970

[15] Tsiolkovsky, K. E., Extension of Man into Outer Space, Proceedings of the Symposium on Jet Propulsion, Vol. 2, United Scientific and Technical Presses, 1936

[16] Tsander, K., From a Scientific Heritage, 1924 (also NASA TTF-541)

[17] McInnes, C. R., and Simmons, J. F. L., "Halo Orbits for Solar Sails: Dynamics and Applications", European Space Agency Journal, Vol. 13, No. 3, pp.229-234, 1989

[18] McInnes, C. R., and Simmons, J. F. L., "Solar Sail Halo Orbits I: Heliocentric Case”, Journal of Spacecraft and Rockets, Vol. 29, No.4, pp. 466-471, 1992

[19] McInnes, C. R., "Passive Control of Displaced Solar Sail Orbits", Journal of Guidance, Control and Dynamics, Vol. 21, No. 6, pp. 975-982, 1998

[20] Hughes, G., and McInnes, C. R., "Solar Sail Hybrid Trajectory Optimisation for Non-Keplerian Orbit Transfer", Journal of Guidance, Control and Dynamics, Vol. 25, No. 3, pp. 602-604, 2002

[21] McInnes, C. R.: “An Inverse Solar Sail Trajectory Problem”, Journal of Guidance, Control and Dynamics, Vol. 26, No. 2, pp. 369-371, 2003

[22] McInnes, C. R., and Simmons, J. F. L., "Solar Sail Halo Orbits II: Geocentric Case”, Journal of Spacecraft and Rockets, Vol. 29, No. 4, pp. 472-479, 1992

[23] Damburg, R. J., and Kolosov, V. V., “A Hydrogen Atom in a Uniform Electric Field”, Journal of Physics B: Atomic, Molecular and Optical Physics, Vol. 9, No. 18, pp. 3149-3157, 1976

[24] Mignard, F., and Henon, M., “About an Unsuspected Integrable Problem”, Celestial Mechanics and Dynamical Astronomy, Vol. 33, No. 3, pp. 239-250, 1984

[25] Dankowicz, H., "Some Special Orbits in the Two-Body Problem with Radiation Pressure", Celestial Mechanics and Dynamical Astronomy, Vol. 58, No.4, pp. 353-370, 1994

[26] Allesandra, C., Benest, D. (Ed.), Froeschlé, C. (Ed.), Singularities in gravitational systems: Applications to chaotic transport in the solar system, Springer-Verlag, Berlin, ISBN 3-540-43765-7, pp. 36, 2002

[27] Molostov, A. A., and Shvartsburg, A. A., "Heliocentric Halos for a Solar Sail with Absorption", Soviet Physics Doklady, Vol. 37, No. 3, pp. 149-152, 1992

[28] Molostov, A. A., and Shvartsburg, A. A., "Heliocentric Synchronous Halos for a Solar Sail with Absorption", Soviet Physics Doklady, Vol. 37, No. 4, pp. 195-197, 1992

[29] Mashkevich, S., V., and Shvartsburg, A. A., "Best Solar Sail for Heliocentric Halos", Soviet Physics Doklady, Vol. 37, No. 6, pp. 290-293, 1992 
[30] Chernikov, Y. A., "The Photogravitational Restricted Three-Body Problem”, Soviet Astronomy, Vol. 14, No. 1, pp 176-181, 1970

[31] Schuerman, D. W., “The Restricted Three-Body Problem Including Radiation Pressure”, The Astrophysical Journal, Vol. 238, pp. 337-342, 1980

[32] Simmons, J. F. L., McDonald, A. J. C., Brown, J. C., "The Restricted Three-Body Problem With Radiation Pressure", Celestial Mechanics and Dynamical Astronomy, Vol. 35, No. 2, pp. 145-187, 1985

[33] Murray, C., "Dynamical Effects of Drag in the Circular Restricted Three-Body Problem", Icarus, Vol. 112, pp. 465-484, 1994

[34] McInnes, C. R., McDonald, A. J. C., Simmons, J. F. L., and MacDonald, E. W., "Solar Sail Parking in Restricted ThreeBody Systems", Journal of Guidance, Dynamics and Control, Vol. 17, No. 2, pp. 399-406, 1994

[35] Forward, R. L., "Statite: A Spacecraft That Does Not Orbit”, Journal of Spacecrafts and Rockets, Vol. 28, No. 5, pp. 606611,1991

[36] McInnes, C. R., “Artificial Lagrange Points for a Partially Reflecting Flat Solar Sail”, Journal of Guidance, Control and Dynamics, Vol. 22, No. 1, pp. 185-187, 1999

[37] McInnes, C. R., Solar Sailing: Technology, Dynamics and Mission Applications, Praxis Publishing, Chichester, ISBN 185233-102-X, pp. 171, 1999

[38] West, J. L., “The GeoStorm Warning Mission: Enhanced Opportunities Based On New Technology", 14 ${ }^{\text {th }}$ AAS/AIAA Spaceflight Mechanics Conference, Paper AAS 04-102, Maui, Hawaii, Feb $8^{\text {th }}-12^{\text {th }}, 2004$

[39] West, J. L., "Solar Sail Vehicle System Design for the GeoStorm Warning Mission”, Structures, Structural Dynamics and Materials Conference, Atlanta, USA, April 2000

[40] McInnes, C. R., "The Existence and Stability of Families of Displaced Two-Body Orbits," Celestial Mechanics and Dynamical Astronomy, Vol. 67, No. 2, 167-180, 1997

[41] McInnes, C. R., "Dynamics, Stability, and Control of Displaced Non-Keplerian Orbits," Journal of Guidance, Control and Dynamics, Vol. 21, No. 5, pp. 799-805, 1998

[42] McInnes, C. R., Solar Sailing: Technology, Dynamics and Mission Applications, Praxis Publishing, Chichester, ISBN 185233-102-X, pp. 196, 1999

[43] McKay, R. J., Macdonald, M., Bosquillon de Frescheville, F., Vasile, M., McInnes, C. R., and Biggs, J. D., "Non-Keplerian Orbits Using Low Thrust, High ISP Propulsion Systems”, In $60^{\text {th }}$ International Astronautical Congress, IAC Paper 09.C1.2.8, Daejeon, Republic of Korea, Oct $12^{\text {th }}-16^{\text {th }}, 2009$

[44] McInnes, C. R., Solar Sailing: Technology, Dynamics and Mission Applications, Praxis Publishing, Chichester, ISBN 185233-102-X, pp. 183, 1999

[45] Xu, M., and Xu, S., "Nonlinear Dynamical Analysis for Displaced Orbits Above a Planet", Celestial Mechanics and Dynamical Astronomy, Vol. 102, pp. 327-353, 2008

[46] McInnes, C. R., "Passive Control of Displaced Solar Sail Orbits", Journal of Guidance, Control, and Dynamics, Vol. 21, No. 6, pp. 975-982, 1998

[47] Bookless, J., and McInnes, C. R., "Dynamics and Control of Displaced Periodic Orbits Using Solar Sail Propulsion", Journal of Guidance, Control, and Dynamics, Vol. 29, No. 3, pp. 527-537, 2006

[48] Gong, S., Baoyin, H., and Li, J., "Relative Orbit Design and Control of Formation Around Displaced Solar Orbits", Aerospace Science and Technology, Vol. 12, No. 2, pp. 195-201, 2008

[49] Lagrange, J.-L., Oeuvres de Lagrange: Essai sur le problème des trios corps, Gallica, Tome 6, pp.272-292, 1772 
[50] Morimoto, M. Y., Yamakawa, H., and Uesugi, K., "Artificial Equilibrium Points in the Low-Thrust Restricted Three-Body Problem", Journal of Guidance, Control, and Dynamics, Vol. 30, No. 5, pp. 1563-1567, 2007

[51] Morimoto, M. Y., Yamakawa, H., and Uesugi, K, "Periodic Orbits with Low-Thrust Propulsion in the Restricted ThreeBody Problem”, Journal of Guidance, Control, and Dynamics, Vol. 29, No. 5, pp. 1131-1139, 2006

[52] Wallace, N., "Testing of the Qinetiq T6 Thruster in Support of the ESA BepiColombo Mercury Mission”, Proceedings of the $4^{\text {th }}$ International Spacecraft Propulsion Conference (ESA SP-555), Chia Laguna, Cagliari, Italy, Jun $2^{\text {nd }}-9^{\text {th }}, 2004$

[53] McInnes, A. I. S, "Strategies for Solar Sail Mission Design in the Circular Restricted Three-Body Problem", M.S. Dissertation, School of Aeronautics and Astronautics, Purdue University, West Lafayette, August, 2000

[54] Lawrence, D., and Piggott, S., "Solar Sailing Trajectory Control for Sub- $\mathrm{L}_{1}$ Stationkeeping”, AIAA Guidance, Navigation and Control Conference and Exhibit, Providence, RI, AIAA Paper 2004-5014, Aug $16^{\text {th }}-19^{\text {th }}, 2004$

[55] Bombardelli, C., and Peláez, J., "On the Stability of Artificial Equilibrium Points in the Circular Restricted Three-Body Problem", Celestial Mechanics and Dynamical Astronomy, DOI: 10.1007/s10569-010-9317-z

[56] Dachwald, B., Mengali, G., Quarta A. A., Macdonald, M., "Parametric Model and Optimal Control of Solar Sails with Optical Degradation”, Journal of Spacecraft and Rockets, Vol. 29, No. 5, pp.1170-1178, 2006

[57] Dachwald, B., Macdonald, M., McInnes, C. R., Mengali, G., Quarta, A. A., "Impact of Optical Degradation on Solar Sail Mission Performance”, Journal of Spacecraft and Rockets, Vol. 44, No. 4, pp. 740-749, 2007

[58] Macdonald, M., and McInnes, C. R., "Solar Sail Mission Applications and Future Advancement", $2^{\text {nd }}$ International Symposium on Solar Sailing, ISSS 2010, New York, USA, July $20^{\text {th }}-22^{\text {nd }}, 2010$

[59] Leipold, M., and, Götz, M., "Hybrid Photonic/Electric Propulsion”, Kayser-Threde, TR SOL4-TR-KTH-0001, Munich, ESA Contract No. 15334/01/NL/PA, January 2002

[60] Mengali, G., and Quarta, A. A., "Trajectory Design with Hybrid Low-Thrust Propulsion System”, Journal of Guidance, Control, and Dynamics, Vol. 30, No. 2, pp. 419-426, 2007

[61] Baig, S., and McInnes, C. R., "Artificial Three-Body Equilibria for Hybrid Low-Thrust Propulsion”, Journal of Guidance, Control, and Dynamics, Vol. 31, No. 6, pp. 1644-1655, 2008

[62] Simo, J., and McInnes, C. R., "Designing Displaced Lunar Orbits Using Low Thrust Propulsion", Journal of Guidance, Control, and Dynamics, Vol. 33, No. 1, pp. 259-265, 2010

[63] Ceriotti, M., and McInnes, C. R., “A Near Term Pole-Sitter Using Hybrid Solar Sail Propulsion”, 2 ${ }^{\text {nd }}$ International Symposium on Solar Sailing, ISSS 2010, New York, USA, July $20^{\text {th }}-22^{\text {nd }}, 2010$

[64] Farquhar, R. W., "The Control and Use of Libration Point Satellites", Ph.D. Dissertation, Department of Aeronautics and Astronautics, Stanford University, Stanford, California, July 1968

[65] Farquhar, R. W., and Kamel, A. A., "Quasi-Periodic Orbits About the Translunar Libration Point", Celestial Mechanics, Vol. 7, pp. 458-473, 1973

[66] Breakwell, J. V, and Brown, J. V., "The 'Halo' Family of 3-Dimensional Periodic Orbits in the Earth-Moon Restricted 3Body Problem", Celestial Mechanics, Vol. 20, pp. 389-404, 1979

[67] Howell, K. C., “Three-dimensional, Periodic, 'Halo' Orbits”, Celestial Mechanics, Vol. 32, pp. 53-71, 1984

[68] Howell, K. C., "Families of Orbits in the Vicinity of the Collinear Libration Points", The Journal of the Astronautical Sciences, Vol. 49, No. 1., pp. 107-125, 2001

[69] Baoyin, H., and McInnes, C. R., "Solar Sail Orbits at the Sun-Earth Artificial L ${ }_{1}$ Point", Celestial Mechanics and Dynamical Astronomy, Vol. 94, No. 2, pp. 155-171, 2006

[70] Waters, T. J., and McInnes, C. R., "Periodic Orbits Above the Ecliptic in the Solar Sail Restricted Three-Body Problem", Journal of Guidance, Control, and Dynamics, Vol. 30, No. 3, pp. 687-693, 2007 
[71] Waters, T. J., and McInnes, C. R., "Invariant Manifolds and Orbit Control in the Solar Sail Three-Body Problem", Journal of Guidance, Control, and Dynamics, Vol. 31, No. 8, pp. 554-562, 2008

[72] Bookless, J., and McInnes, C. R., "Control of Lagrange Point Orbits Using Solar Sail Propulsion”, Acta Astronautica, Vol. 62, No. 2-3, pp. 159-176, 2008

[73] Baig, S., and McInnes, C. R., "Artificial Halo Orbits for Low-Thrust Propulsion Spacecraft", Celestial Mechanics and Dynamical Astronomy, Vol. 104, No. 4, pp. 321-335, 2009

[74] Farrés, A., and Jorba À, "Periodic and Quasi-periodic Motions of a Solar Sail Close to $S \mathrm{~L}_{1}$ in the Earth-Sun System", Celestial Mechanics and Dynamical Astronomy, Vol. 107, pp. 233-253, 2010

[75] Belbruno, E., and Miller J., "A Ballistic Lunar Capture Trajectory for the Japanese Spacecraft Hiten”, Jet Propulsion Laboratory Interoffice Memorandum, 312/90.4-1317, 1990

[76] Belbruno, E., Advances in Astrophysics Volume 1, Chapter 4: Low-energy transfers and applications, Elsevier Astrodynamics Series, Butterworth-Heinemann, ISBN 9790123735621, pp. 107-126, 2007

[77] Koon, W. S., Lo, M. W., Mardsen, J. E., and Ross, S. D., "Heteroclinic Connections Between Periodic Orbits and Resonance Transitions in Celestial Mechanics", Chaos, Vol. 10, No. 2, pp. 427-469, 2000

[78] Gómez, G., Koon, W. S., Lo, M. W., Mardesn, J. E., Masdemont, J., and Ross, S. D., "Connecting Orbits and Invariant Manifolds in the Spatial Restricted Three-Body Problem”, Nonlinearity, Vol. 17, No. 5, pp. 1571-1606, 2004

[79] Howell, K. C., and Kakoi, M., "Transfers Between the Earth-Moon System and Sun-Earth Systems Using Manifolds and Transit Orbits", Acta Astronautica, Vol. 59, pp.367-380, 2006

[80] Howell, K. C., Beckman, M., Patterson, C., and Folta, D., "Representations of Invariant Manifolds for Applications in Three-Body Systems", The Journal of the Astronautical Sciences, Vol. 54, No. 1, pp. 69-93, 2006

[81] Pergola, P., Geurts, K., Casaregola, C., and Andrenucci, M, "Earth-Mars Halo to Halo Low Thrust Manifold Transfers", Celestial Mechanics and Dynamical Astronomy, Vol. 105, No. 1-3, pp. 19-32, 2009

[82] Gong, S., Baoyin, H, and Li, J., "Solar Sail Three-Body Transfer Trajectory Design", Journal of Guidance, Control, and Dynamics, Vol. 33, No. 3, pp. 873-886, 2010

[83] Waters, T. J., and McInnes, C. R., "Solar Sail Dynamics in the Three-Body Problem; Homoclinic Paths of Points and Orbits", International Journal of Non-Linear Mechanics, Vol. 43, pp. 490-496, 2008

[84] Farrés, A., and Jorba, À, "Solar Sail Surfing Along Families of Equilibrium Points", Acta Astronautica, Vol. 63, pp. 249257,2008

[85] Janhunen, P., "Electric Sail for Spacecraft Propulsion", AIAA Journal of Propulsion and Power, Vol. 20, No. 4, pp. 763-764, 2004

[86] Janhunen, P., and Sandroos, A., "Simulation Study of Solar Wind Push on a Charged Wire: Basis of Solar Wind Electric Sail Propulsion”, Annales Geophysicae, Vol. 25, No. 3, pp. 755-767, 2007

[87] Mengali, G., and Quarta, A. A., "Non-Keplerian Orbits for Electric Sails", Celestial Mechanics and Dynamical Astronomy, Vol. 105, No. 1-3, pp. 179-195, 2009

[88] Forward, R. L., "Solar Photon Thruster", Journal of Spacecraft and Rockets, Vol. 27, No. 4, pp. 411- 416, 1990

[89] Mengali, G. and Quarta, A.A., "Compound Solar Sail with Optical Properties: Models and Performance," Journal of Spacecraft and Rockets, Vol. 43, No. 1, pp. 239-245, 2006

[90] B. Dachwald, P. Wurm, "Design Concept and Modeling of an Advanced Solar Photon Thruster", $19^{\text {th }}$ AAS/AIAA Space Flight Mechanics Meeting, Paper AAS 09-147, Savannah, Georgia, February 2009

[91] Matloff, G. L., "The Solar Photon Thruster as a Terrestrial Pole Sitter", Annals of the New York Academy of Sciences, Vol. 1017, pp. 468-474, 2004 
[92] Peck, M. A., "Prospects and Challenges for Lorentz-Augmented Orbits," AIAA Guidance, Navigation, and Control Conference, AIAA Paper 2005-5995, San Francisco, United States, August 2005

[93] Streetman, B., and Peck, M. A., "New Synchronous Orbits Using the Geomagnetic Lorentz Force", Journal of Guidance, Control, and Dynamics, Vol. 30, No. 6, pp.1677-1690, 2007

[94] Andrews, D. G., and Zubrin, R. M., "Magnetic Sails and Interstellar Travel," 39th Congress of the International Astronautical Federation, IAF-88-553, Bangelore, India, October 1988

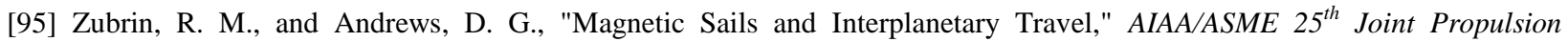
Conference, AIAA-89-2441, Monterey, CA, July 1989

[96] Love, S. G., and Andrews, D. G., "Applications of Magnetic Sails," 42nd Congress of the International Astronautical Federation, IAF 91-245, Montreal, Canada, October 1991

[97] Zubrin, R., "The Use of Magnetic Sails to Escape from Low Earth Orbit," Journal of the British Interplanetary Society, Vol. 46, pp.3-10, 1993

[98] Winglee, R. M., Slough, J., Ziemba, T., and Goodson, A., "Mini-magnetospheric plasma propulsion: tapping the energy of the solar wind for spacecraft propulsion”, Journal of Geophysical Research, Vol. 105, pp. 21067-21077, 2000

[99] Kajimura, Y., Shinohara, D., Noda, K., and Nakashima, H., "Numerical Simulation of a Magneto Plasma Sail by Using Three-dimensional Hybrid Code”, Journal of Plasma Physics, Vol. 72, part 6, pp. 877-881, 2006

[100] McInnes, C. R., "Solar Sail Trajectories at the Lunar L L Lagrange Point”, Journal of Spacecraft and Rockets, Vol. 30, No. 6, pp. 782-784, 1993

[101] Vonbun, F. O., “A Hummingbird for the Lunar $\mathrm{L}_{2}$ Libration Point”, NASA TN D-4468, 1968

[102] Simo, J., and McInnes, C. R., "Solar Sail Trajectories at the Earth-Moon Lagrange Points", In 59 ${ }^{\text {th }}$ International Astronautical Congress, IAC Paper 08.C1.3.13, Glasgow, Scotland, Sep $29^{\text {th }}-$ Oct $3^{\text {rd }}, 2008$

[103] Simo, J., and McInnes, C. R., "Solar Sail Orbits at the Earth-Moon Libration Points", Communications in Nonlinear Science and Numerical Simulation, Vol. 14, No. 12, pp. 4191-4196, 2009

[104] Simo, J., and McInnes, C. R., “Asymptotic Analysis of Displaced Lunar Orbits”, Journal of Guidance, Control, and Dynamics, Vol. 32, No. 5, pp. 1666-1671, 2009.

[105] Wawrzyniak, G. G., and Howell, K. C., "The Solar Sail Lunar Relay Station: An Application of Solar Sails in the EarthMoon System", In 59 $9^{\text {th }}$ International Astronautical Congress, IAC Paper 08.C1.3.14, Glasgow, Scotland, Sep $29^{\text {th }}-$ Oct $3^{\text {rd }}$, 2008 [106] Ozimek, M. T., Grebow, D. J., Howell, K. C., "Designs of Solar Sail Trajectories with Applications to Lunar South Pole Coverage", Journal of Guidance, Control, and Dynamics, Vol. 32, No.6, pp. 1184-1197, 2009

[107] Baoyin, H., and McInnes, C. R., "Solar Sail Equilibria in the Elliptical Restricted Three-Body Problem", Journal of Guidance, Control, and Dynamics, Vol. 29, No. 3, pp. 538-543, 2006

[108] Biggs, J. D., McInnes, C. R., and Waters, T., "Control of Solar Sail Periodic Orbits in the Elliptic Three-Body Problem", Journal of Guidance, Control, and Dynamics, Vol. 32, No. 1, pp. 318-320, 2009

[109] Howell, K. C., and Spencer, D. B., "Periodic Orbits in the Restricted Four-Body Problem", Acta Astronautica, Vol. 13, No. 8, pp. 473-479, 1986

[110] Ceccaroni, M., Biggs, J., and McInnes, C. R., "Extension of Low-Thrust Propulsion to the Autonomous Coplanar Circular Restricted Four Body Problem with Application to Future Trojan Asteroid Missions", In 61 ${ }^{\text {st }}$ International Astronautical Congress, IAC Paper 10.C1.1.2, Prague, Czech Republic, Sep $27^{\text {th }}$ - Oct $1^{\text {st }}, 2010$

[111] Dittberner, G. J., Crison, M., Bajpai, S., and Diedrich, B., "Advanced Technologies for Future Environmental Satellite Systems”, 2004 EUMETSAT Meteorological Satellite Conference, Prague, Czech Republic, May $31^{\text {st }}-\mathrm{June} 4^{\text {th }}, 2004$ 
[112] Diedrich, B., and Mulligan, P., "Solar Sails and Artificial Lagrange Orbits for Remote Sensing, Telecommunications, and Space Weather Applications", 87 $7^{\text {th }}$ American Meteorological Society Annual Meeting, Third Symposium on Future National Operational Environmental Satellites, San Antonio, United States, Jan $13^{\text {th }}-18^{\text {th }}, 2007$

[113] Baig, S., and McInnes, C. R., "Light-Levitated Geostationary Orbits Are Feasible", Journal of Guidance, Control, and Dynamics, Vol. 33, No. 3, pp.782-793, 2010.

[114] McKay, R. J., Macdonald, M., Vasile, M., and Bosquillon de Frescheville, F., “A Novel Interplanetary Communications Relay”, In AIAA/AAS Astrodynamics Specialist Conference, Toronto, Canada, Aug $2^{\text {nd }}-5^{\text {th }}, 2010$

[115] Grebow, D., Ozimek, M., and Howell, K., "Design of Optimal Low-Thrust Lunar Pole-Sitter Missions", AAS/AIAA Space Flight Mechanics Meeting, Savannah, Georgia, AAS 09-148, February 2009

[116] Grebow, D., Ozimek, M., and Howell, K., “Advanced Modeling of Optimal Low-Thrust Pole-Sitter Trajectories”, In 60 International Astronautical Congress, IAC Paper 09.C1.5.4, Daejeon, Republic of Korea, Oct $12^{\text {th }}-16^{\text {th }}, 2009$

[117] Grebow, D. J., Ozimek, M. T., Howell, K. C., and Folta, D. C., "Multibody Orbit Architectures for Lunar South Pole Coverage", Journal of Spacecraft and Rockets, Vol. 45, No. 2, pp. 344-358, 2008

[118] McInnes, C. R., “Solar Sailing: Mission Applications and Engineering Challenges”, Philosophical Transactions of the Royal Society A, Vol. 361, No. 1813, pp. 2989-3008, 2003

[119] Spilker, T. R., “Saturn Ring Observer”, Acta Astronautica, Vol. 52, pp. 259-265, 2003

[120] Sawai, S., Scheeres, D. J., and Broschart, S, "Control of Hovering Spacecraft using Altimetry," Journal of Guidance, Control, and Dynamics, Vol. 25, No. 4, pp. 786-795, 2002

[121] Broschart, S. B., and Sheeres, D. J., "Control of Hovering Spacecraft Near Small Bodies: Application to Asteroid 25143 Itokawa”, Journal of Guidance, Control, and Dynamics, Vol. 28, No. 2, pp. 343-354, 2005

[122] Broschart, S. B., and Scheeres, D. J., "Boundedness of Spacecraft Hovering Under Dead-Band Control in Time-Invariant Systems," Journal of Guidance, Control and Dynamics, Vol. 30, No. 2, pp. 601-610, 2007

[123] Morrow, E., Scheeres, D. J., and Lubin, D., "Solar Sail Orbit Operations at Asteroids", Journal of Spacecraft and Rockets, Vol. 38, No. 2, pp. 279-286, 2001

[124] Sauer Jr., C. G., “The L Liamond Affair”, Spaceflight Mechanics Conference, AAS 04-278, Maui, Hawaii, February 2004

[125] Biggs, J. D., and McInnes, C. R., "Solar Sail Formation Flying for Deep-Space Remote Sensing”, Journal of Spacecraft and Rockets, Vol. 46, No. 3, pp. 670-678, 2009

[126] Kezerashvili, R. Ya., and Vázquez-Poritz, J. F., "Solar Radiation Pressure and Deviations from Keplerian Orbits for Solar Sails", Physics Letters B, Vol. 675, Issue 1, pp. 18-21, 2009

[127] Kezerashvili, R. Ya., and Vázquez-Poritz, J. F., "Bound Orbits of Solar Sails and General Relativity", Advances in Space Research, Vol. 46, Issue 3, pp. 346-361, 2010

[128] McInnes, C. R., "Space-Based Geoengineering: Challenges and Requirements", Proceedings of the Institution of Mechanical Engineers Vol. 223, Part C: Journal of Mechanical Engineering Science, JMES1439, September 2009

[129] Early, J. T., "Space-Based Solar Shield to Offset Greenhouse Effect”, Journal of the British Interplanetary Society, Vol. 42, pp. 567-569, 1989

[130] Sefritz, W., “Mirrors to Halt Global Warming?”, Nature, Vol. 340, No. 603, 24 August, 1989

[131] Roy, K. I., and Kennedy, R., "Mirrors and Smoke - Ameliorating Climate Change with Giant Solar Sails", Whole Earth Review, Summer 2001

[132] Govindasamy, B., and Caldeira, K., "Geoengineering Earth's Radiation Balance to Mitigate $\mathrm{CO}_{2}$-Induced Climate Change", Geophysical Research Letters, Vol. 27, No. 14, pp. 2141-2144, 2000 
[133] Teller, E., and Hyde, R., "Global Warming and Ice Ages: 1. Prospects for Physics-Based Modulation of Global Change", $22^{\text {nd }}$ International Seminar on Planetary Emergencies, Erice, Sicily, LLNL preprint UCRL-JC128715, $20-23$ August, 1997

[134] McInnes, C. R., “Minimum Mass Solar Shield for Terrestrial Climate Control”, Journal of the British Interplanetary Society, Vol. 55, pp. 307-311, 2002

[135] McInnes, C. R., "Planetary Macro-Engineering Using Orbiting Solar Reflectors", In Macro-engineering: a challenge for the future (Springer, Berlin), pp. 215-250, 2006

[136] Angel, R., "Feasibility of Cooling the Earth with a Cloud of Small Spacecraft Near the Inner Lagrange Point $\left(\mathrm{L}_{1}\right)$ ", Proceedings of the National Academy of Sciences, Vol. 103, No. 46, pp. 17184-17189, 2006

[137] Biggs, J. D., and McInnes, C. R., "Passive Control for Space-Based Geo-Engineering”, Journal of Guidance, Control, and Dynamics, Vol. 33, No. 3, May-June 2010 